\title{
Long-read 16S-seq reveals nasopharynx microbial dysbiosis and enrichment of Mycobacterium and Mycoplasma in COVID-19 patients: A source of co- infection
}

Punit Prasad ( $\sim$ punit@ils.res.in )

Institute of Life Sciences https://orcid.org/0000-0002-9132-6078

Soumendu Mahapatra

Institute of Life Sciences

Rasmita Mishra

Institute of Life Sciences

Krushna Chandra Murmu

Institute of Life Sciences

Shifu Aggarwal

Institute of Life Sciences

Manisha Sethi

Institute of Life Sciences

Priyanka Mohapatra

Institute of Life Sciences

Arup Ghosh

Institute of Life Sciences

Rina Yadav

Institute of Life Sciences

Hiren Dodia

Institute of Life Sciences

Shamima Azma Ansari

Institute of Life Sciences

Saikat De

Institute of Life Sciences

Deepak Singh

Institute of Life Sciences

Amol Suryawanshi

Institute of Life Sciences

Rupesh Dash

Institute of Life Sciences 


\section{Shantibhushan Senapati}

Institute of Life Sciences

Tushar K. Beuria

Institute of Life Sciences

Soma Chattopadhyay

Institute of Life Sciences

Gulam Hussain Syed

Institute of Life Sciences

Rajeeb Swain

Institute of Life Sciences

Sunil K. Raghav

Institute of Life Sciences

Ajay Parida

Institute of Life Sciences

\section{Research Article}

Keywords: Nasopharyngeal microbiome, Nanopore, COVID-19, 16S rRNA, Mycobacterium and Mycoplasma

Posted Date: December 22nd, 2021

DOI: https://doi.org/10.21203/rs.3.rs-1149233/v1

License: (c) (i) This work is licensed under a Creative Commons Attribution 4.0 International License. Read Full License 


\section{Long-read 16S-seq reveals nasopharynx microbial dysbiosis and enrichment of}

\section{Mycobacterium and Mycoplasma in COVID-19 patients: A source of co-infection}

Punit Prasad $^{1 * \$}$, Soumendu Mahapatra $^{1 *}$, Rasmita Mishra ${ }^{1 *}$, Krushna Chandra Murmu $^{1}$, Shifu Aggarwal $^{1}$, Manisha Sethi ${ }^{1}$, Priyanka Mohapatra ${ }^{1}$, Arup Ghosh ${ }^{1}$, Rina Yadav ${ }^{1}$, Hiren Dodia ${ }^{1}$, Shamima Azma Ansari ${ }^{1}$, Saikat De ${ }^{1}$, Deepak Singh ${ }^{1}$, Amol Suryawanshi ${ }^{1}$, Rupesh Dash ${ }^{1}$, Shantibhushan Senapati ${ }^{1}$, Tushar K. Beuria ${ }^{1}$, Soma Chattopadhyay ${ }^{1}$, Gulam Hussain Syed ${ }^{1}$, Rajeeb Swain ${ }^{1}$, Sunil K. Raghav ${ }^{1}$, Ajay Parida ${ }^{1 \$}$

${ }^{1}$ Institute of Life Sciences, Bhubaneswar, Odisha, India.

* These authors contributed equally to this work

$\$$ Correspondence: Ajay Parida, Ph.D.

Institute of Life Sciences,

Nalco Square, Chandrasekharpur,

Bhubaneswar, Odisha -751023

Phone: +91-674-2304324

Email: ajayparida@ils.res.in drajayparida@gmail.com

\section{Punit Prasad, Ph.D.}

Institute of Life Sciences,

Nalco Square, Chandrasekharpur,

Bhubaneswar, Odisha - 751023

Phone: +91-674-2304319

Email: punit@ils.res.in punit.ils@gov.in

Running title: 16S rRNA-sequencing of nasopharyngeal microbiome identifies Mycobacterium and Mycoplasma in COVID-19 patients

Keywords: Nasopharyngeal microbiome, Nanopore, COVID-19, 16S rRNA, Mycobacterium and Mycoplasma 


\section{$1 \quad$ Abstract}

\section{$2 \quad$ Background}

3 The coronavirus disease 2019 (COVID-19) pandemic caused by severe acute respiratory 4 syndrome corona virus 2 (SARS-CoV-2) is a major global health concern. This virus infects

5 the upper respiratory tract and causes pneumonia-like symptoms. So far, few studies have

6 shown alterations in nasopharyngeal (NP) microbial diversity, enrichment of opportunistic

7 pathogens and their role in co-infections during respiratory infections. Therefore, we

8 hypothesized that microbial diversity changes, with increase in the population of opportunistic

9 pathogens, during SARS-CoV2 infection in the nasopharynx which may be involved in co-

10 infection in COVID-19 patients.

\section{$11 \quad$ Methods}

12 The 16S rRNA variable regions, V1-V9, of NP samples of control and COVID-19 13 (symptomatic and asymptomatic) patients were sequenced using the Oxford Nanopore ${ }^{\mathrm{TM}}$ 14 technology. Comprehensive bioinformatics analysis for determining alpha/beta diversities, 15 non-metric multidimensional scaling, correlation studies, canonical correspondence analysis, 16 linear discriminate analysis, and dysbiosis index were used to analyze the control and COVID17 19-specific NP microbiomes.

\section{$18 \quad$ Results}

19 We observed significant dysbiosis in COVID-19 NP microbiome with increase in abundance 20 of opportunistic pathogens at genus and species levels in asymptomatic/symptomatic patients.

21 The significant abundance of Mycobacteria spp. and Mycoplasma spp. in symptomatic 22 patients suggest their association and role in co-infections in COVID-19 patients. 
23 Furthermore, we found strong correlation of enrichment of Mycobacteria and Mycoplasma

24 with the occurrences of chest pain and fever in symptomatic COVID-19 patients.

\section{Conclusion}

26 This is the first study from India to show the abundance of Mycobacteria and Mycoplasma

27 opportunistic pathogens in non-hospitalized COVID-19 patients and their relationship with 28 symptoms, indicating the possibility of co-infections.

\section{$30 \quad$ Introduction}

31 The coronavirus disease 2019 (COVID-19) pandemic, a global health threat, is caused by the 32 severe acute respiratory syndrome coronavirus 2 (SARS-CoV-2). The symptoms range from 33 fever, throat pain, and loss of taste and smell to severe congestion in the chest, drop in oxygen 34 levels, pneumonia, and acute respiratory distress syndrome (1). A significant population 35 worldwide has been found to be asymptomatic and to act as spreaders of the infection (2). The 36 virus enters the host via the upper respiratory tract (URT) where the spike protein binds to the 37 angiotensin I converting enzyme 2 (ACE2) receptor, an essential step in invading host cells to 38 cause progressive disease $(3,4)$. Random mutations in the SARS-CoV2 spike protein and 39 receptor-binding domain promote efficient invasion and enhance pathogenicity (5).

40 The nasopharyngeal (NP) tract is inhabited by a large number of microbial communities which

41 maintain normal homeostasis (6). Studies have revealed association between microbial 42 communities that influence viral infections of the lung, such as chronic rhinosinusitis, asthma, 43 pneumonia, and cystic fibrosis in the URT $(7,8)$. URT infections lead to decrease in microbial 44 diversity, resulting in dysbiosis, which potentiate increase in the opportunistic pathogen 45 population and promote the growth of other pathogens in immunocompromised hosts $(9,10)$. 
46 Reports have shown that NP swabs in virus transport media can be used to investigate the NP

47 microbial composition in COVID-19 patients $(11,12)$. Recent studies have revealed overall

48 compositional changes in the NP microbiota and increase in the number of opportunistic

49 pathogens such as Rothia and Veillonella in COVID-19 patients with shortness of breath (11,

$5013,14)$. Secondary infections in patients with COVID-19 are associated with the abundance

51 of opportunistic pathogens such as Moraxella, Corynebacterium, Haemophilus,

52 Stenotrophomonas, Acinetobacter, Fusobacterium periodonticum, Mycobacterium spp.,

$53 \quad$ Mycoplasma pneumonia and Pseudomonas aeruginosa (15-19).

54 The treatment modalities of SARS-CoV2 infection are not yet established and patients are 55 administered immunosuppressants to regulate cytokine storm, thereby creating an 56 environment conducive for the growth of opportunistic pathogens and co-infections in 57 COVID-19 patients $(19,20)$. Several case reports have highlighted fungal, viral, and bacterial 58 co-infections in COVID-19 patients with severe symptoms (19). However, information 59 regarding the crosstalk between SARS-CoV-2 infection and NP microbiota is scarce. The 60 absence of an animal model makes it difficult to test and validate the role of NP microbiota in 61 SARS-CoV-2 infection. Various studies so far have shown differences in the abundance of 62 various opportunistic pathogens in the NP microbiota of patients, which is one of the 63 bottlenecks in this area of research. Hence more studies on the NP microbiome are required 64 for understanding its role in COVID-19 patients and its relation with symptom severity, as 65 microbial composition is dynamic and changes with multiple extrinsic factors.

66 Here, we hypothesized that similar to other respiratory diseases (asthma, pneumonia, and 67 cystic fibrosis) dysbiosis might occur in the COVID-19 NP microbial community (high virus68 bacteria interaction zone), resulting in emergence of opportunistic pathogens, which may be 
associated with symptoms of COVID-19. In this regard, we also predicted that the alterations in NP microbiota may occur in both symptomatic and asymptomatic COVID-19-patients and that the microbial composition may differ between these two categories of patients, studies on

72 which are limited. In this study, we have investigated the alterations in the NP microbial 73 community of patients with active COVID-19 $(n=46)$ and compared them with those of the 74 healthy individuals $(\mathrm{n}=12)$. We also analyzed the differences in the microbial community 75 composition between asymptomatic $(n=25)$ and symptomatic $(n=21)$ COVID-19 patients.

76 We have used the metataxonomic approach of 16S rRNA long-read sequencing (V1-V9) with 77 the Nanopore sequencing method to elucidate the reduction in microbial diversity in patients 78 with COVID-19. We found enrichment of Mycobacteria and Mycoplasma in both 79 asymptomatic and symptomatic patients with COVID-19, however, the abundance in 80 symptomatic patients were much higher and it strongly correlated with chest pain and fever.

\section{$81 \quad$ Materials and Methods}

82 Ethical approval: Ethical permission for nasopharyngeal microbiome study and the 83 biorepository was obtained from the Institutional Ethical Committee (IEC)/Institutional 84 Review Board (IRB) of the Institute of Life Sciences [(102/HEC/2020) and (100/HEC/2020)]. 85 Approval was also obtained from the Institutional Biosafety Committee (IBSC) (V-12286

87 from the Review Committee on Genetic Manipulations (RCGM) under Department of 88 Biotechnology, Ministry of Science and Technology.

89 Sample collection and reverse transcription-polymerase chain reaction (RT-PCR): In 90 total, $60 \mathrm{NP}$ samples were collected for $16 \mathrm{~S}$ rDNA amplicon sequencing from the Institute of 91 Life Science (ILS) COVID-19 sample biorepository unit. The COVID-19-positive samples (n 
$92=47$ ) were confirmed by amplifying the genes encoding SARS-CoV-2 nucleocapsid, spike, 93 and ORF1ab/RdRP using either TaqPath ${ }^{\mathrm{TM}}$ COVID-19 combo kit (Invitrogen, A47814) or 94 Meril COVID-19 one-step RT-PCR kit (Meril Diagnostics, NCVPCR-02). All samples were 95 collected in the hospital setup prior to the medication. These patients were not treated with 96 antibiotics as they were not aware of their COVID-19 test results. The patients were grouped 97 as symptomatic $(\mathrm{n}=22)$ or asymptomatic $(\mathrm{n}=25)$ based on their clinical data. The control 98 samples $(\mathrm{n}=13)$ were negative for SARS-CoV-2 virus RNA, and none of the subjects from 99 whom the samples were obtained had any flu-like symptoms. All samples were collected in 100 viral transport media (VTM) and stored at $-80 \square \mathrm{C}$ until DNA isolation.

101 DNA extraction and PCR amplification: DNA was isolated using the PureLink ${ }^{\mathrm{TM}}$ 102 microbiome DNA purification kit (Invitrogen, A29790) according to the manufacturer's 103 protocol and eluted in $40 \mu \mathrm{l}$ elution buffer. The quality and quantity of DNA were determined 104 using the Multiskan ${ }^{\mathrm{TM} G O}$ spectrophotometer (Thermo Scientific).

$10516 \mathrm{~S}$ rDNA amplification, library preparation, and sequencing: The V1-V9 variable regions of 106 the $16 \mathrm{~S}$ rRNA gene were amplified using 130-F (5'107 GGCGGATCCAAGGAGGTGTTCCAGCCGC-3') and 139-R (5'108 GGCCTCGAGAGAGTTTGATCCTGGCTCAGG-3') primers. PCR $(50 \mu \mathrm{l})$ was set up using 109 total DNA (10 ng) isolated from NP samples, primers (5 nM), and NEB Q5® High-Fidelity 2 $110 \mathrm{X}$ master mix (NEB, M0492L) per the manufacturer's protocol. The amplicons ( $\sim 1.6 \mathrm{~kb})$ were 111 analyzed on $0.8 \%$ agarose gel and cleaned using DNA Clean and Concentrator-25 kit (Zymo 112 Research, D4034). The PCR products were quantified using a Qubit 4 fluorometer (Thermo 113 Scientific) using the Qubit ${ }^{\circledR}$ dsDNA BR assay kit (Thermo Scientific, Q32853). Amplicon 114 libraries were generated following the Oxford Nanopore 1D library preparation protocol using 
the PCR barcoding (96) genomic DNA kit (Oxford Nanopore ${ }^{\mathrm{TM}}$, SQK-LSK109). Equimolar amounts of amplicon libraries were pooled and sequenced using the MinION OXFORD NANOPORETM device at the ILS DNA sequencing facility.

Microbiome data processing: RAW fast5 files were generated using the MinKNOWTM tool for individual samples. Base calling was performed using the Guppy base-caller and fastq files were generated. FastQC of each sample was performed using the Babraham fastqc suite (https://www.bioinformatics.babraham.ac.uk/projects/fastqc/), followed by trimming of low quality reads using nanoflit. Operational taxonomic units (OTUs) were generated using Kraken2 (https://ccb.jhu.edu/software/kraken2/index.shtml) (21) and the unclassified reads were filtered for downstream analysis using the 'phyloseq' ' $R$ " package to generate combined OTUs for all the samples and metadata (Supplemental Table 1). Read counts for mitochondria and chloroplast were discarded. Normalization and differential OTU abundance were determined between control, and symptomatic and asymptomatic subjects using the DESeq2 function (cutoff of p-value $\leq 0.05)(22)$.

\section{In-depth microbiome data analysis:}

Diversity analysis: Alpha diversity was assessed using the Shannon and Simpson diversity indices. Statistical significance was estimated using the Wilcoxon rank sum test. The beta diversity significance among groups was examined using PERMANOVA (p-value 0.001). Ordination analysis was performed using principal coordinate analysis (PCoA), non-metric multidimensional scaling (NMDS) and canonical correspondence analysis (CCA). R packages, 'microbiome','Vegan','ade4', and ggpubr, were used for analysis and 'ggplot2' was used for visualization. 
137 Dysbiosis index: Microbiome dysbiosis in each sample was calculated based on Bray-Curtis 138 distances. All samples were subjected to PCoA using Bray-Curtis distances. Next, the centroid 139 (median) of the control subjects was calculated along PCoA axes. The dysbiosis score for each 140 sample was calculated as a Euclidian distance between its position in the PCoA space and 141 control centroid $\left(\mathrm{DI}(\mathrm{X}, \mathrm{HC})=\sqrt{(\mathrm{X} i-H C i)^{2}+(\mathrm{X} j-H C j)^{2}} \mid(\mathrm{DI}\right.$ : Dysbiosis Index, X: Samples, 142 HC: Control Centroid). Their significance was assessed using Wilcoxon and Kruskal-Wallis 143 test (23).

144 Sample correlation: Correlation matrix between samples and OTUs for each taxonomic level 145 (phylum, order, family, and genus) from differential OTUs was obtained using Spearman's 146 correlation method and it was visualized as a heat map. Correlation coefficients for each 147 sample correlation pair and each classification level and density plot were plotted with mean 148 and median. The Kolmogorov-Smirnov test (KS) was used to determine the significance in 149 sample groups (control, asymptomatic, and symptomatic).

150 Linear discriminant analysis (LDA) effect size (LEfSe) analysis: The LEfSe was calculated 151 using the online Galaxy web application and the Huttenhower lab's tool 152 (https://huttenhower.sph.harvard.edu/galaxy/). LDA effect size was calculated using the 153 Kruskal-Wallis sum rank test $(\mathrm{p}$-value $\leq 0.05)$ and it detected differential abundant features at 154 genus and species level within three sample groups. Significance at the taxonomic level was 155 then tested using the pairwise Wilcoxon rank-sum tests ( $p$-value $\leq 0.05)$. Finally, the effect 156 size of each differentially abundant feature was estimated using LDA. One-against-all sample 157 groups were compared and a linear discriminant analysis score greater than 3.6 was set as the 158 threshold; all-against-all sample groups were compared and a linear discriminant analysis 
score greater than 2.0 was set as the threshold. Cladogram was used for identification of taxa

160 at different levels of the taxonomic hierarchy between sample groups (LDA score > 2).

161 Network analysis: Network was constructed using weighted correlation network analysis or 162 weighted gene co-expression network analysis (WGCNA) (24). Briefly, pairwise Spearman 163 correlation between OTUs (which was generated from LefSe analysis) was calculated using 164 the WGCNA function. Network metrics such as betweenness, closeness, Eigen centrality, and 165 PageRank centrality of the resulting network were calculated and visualized using 'Gephi', 166 (https://gephi.org/) (25).

$168 \quad$ Results

169 Study design and subject attributes

170 The role of the microbiome in viral infections is an emerging field and we were interested in 171 understanding NP microbial diversity and changes in its composition in patients during SARS172 CoV-2 infection. We collected NP samples from COVID-19 patients between 11th May 2020 173 and 10th October 2020 to study alterations in the NP microbiome. The schematic 174 representation of the study is shown in Figure 1A. In total, 60 NP samples subjects (infected, $175 \mathrm{n}=47$ and control, $\mathrm{n}=13$ subjects, positive and negative for SARS-CoV2 RT-qPCR test 176 respectively) were obtained from the Institute of Life Sciences biorepository. Out of 47 SARS177 CoV-2-positive subjects, 25 were asymptomatic and 22 were symptomatic with mild 178 symptoms (Figure 1B). In total, 179, 59, 691 reads were generated. Two samples with low 179 read counts (1 from control and other from symptomatic category) were excluded and the final 180 study was performed with 58 subjects, including the control (C) [n = $12(21 \%)]$, and 181 asymptomatic [IA, infected asymptomatic; $\mathrm{n}=25(43 \%)$ ] and symptomatic patients [IS, 
182 infected symptomatic; $\mathrm{n}=21(36 \%)$ ]. The details of the participants considered for this study

183 are shown in Table 1. Differential OTUs $(\mathrm{n}=795, \mathrm{p} \leq 0.05)$ were obtained from 3482 OTUs

184 using the deseq2 function by comparing with control NP subjects. For downstream analysis

185 differential, 795 OTUs were considered. We used the t-distributed stochastic neighbor

186 embedding ( $\mathrm{t}-\mathrm{SNE}$ ) dimension reduction method to obtain the overall distribution of NP

187 samples with 795 OTUs (Figure 1C). We found that the control and SARS-CoV-2-infected

188 subjects showed distinct segregation of OTUs in the NP microbiome, while asymptomatic and

189 symptomatic subjects showed modest separation. This indicated that the abundance of the 795

190 differential OTUs potentially determines the compositional distribution patterns.

\section{NP microbiome diversity was significantly altered in SARS-CoV-2-infected patients}

192 Distinct distribution of OTUs from control and infected patients prompted us to compare the 193 alpha diversities (evenness and richness of bacterial community compositions) using Shannon 194 and Simpson indices. The Shannon and Simpson microbial diversity indices differed 195 significantly between control and SARS-CoV-2-infected participants ( $\mathrm{p}$-value $\leq 0.05$ ) in 196 pairwise Wilcoxon rank test (Shannon $\mathrm{p}$-value $=3.0 * 10^{-4}$ and Simpson $\mathrm{p}$-value $=3.3 * 10^{-3}$ ) 197 (Figure 2A, B). Although the alpha diversity indices for samples from symptomatic and 198 asymptomatic patients compared to control subjects were found to be significantly reduced, 199 no difference was observed between symptomatic and asymptomatic samples (Figure 2C, D). 200 Furthermore, we used a linear regression model to establish the association between total OTU 201 read counts for each sample and Shannon/Simpson alpha diversity indices. We found negative 202 correlation for both Shannon (IA - R $=-0.35, \mathrm{R}^{2}=0.44, \mathrm{p}=0.083$; IS - R $=-0.54, \mathrm{R}^{2}=0.48$, $203 \mathrm{p}=0.012$ ) and Simpson (IA $-\mathrm{R}=-0.58, \mathrm{R}^{2}=0.68, \mathrm{p}=0.0028 ; \mathrm{IS}-\mathrm{R}=-0.77, \mathrm{R}^{2}=0.63, \mathrm{p}=$ $2047.7 * 10^{-5}$ ) alpha diversity indices with $95 \%$ confidence intervals with total OTU counts (Figure 
2E, F). To further understand the microbial composition dissimilarity within the samples, we analyzed beta diversity using PCoA and applied both unweighted (microbial richness) and

207 weighted (microbial richness and abundance) unifrac distance methods. The first two 208 components of PCoA showed $60.3 \%$ and $80.1 \%$ variance for the unweighted and weighted 209 unifrac method. The overall difference in microbial population showed two different clusters 210 of control and SARS-CoV-2-infected patients (IA and IS) in the unifrac weighted method, 211 while the unifrac unweighted method showed more clear segregation between symptomatic 212 and asymptomatic samples (Figure 2G). We assessed the significance of beta diversity to 213 calculate unifrac distance matrix (PERMANOVA test with 999 permutations) for both 214 unweighted and weighted methods and found that the three sample groups (C, IA, and IS) 215 differed significantly $(\mathrm{P}=0.001)$ with $18 \%$ variance explained $\left(\mathrm{R}^{2}=0.18842\right)$.

\section{NP microbiome dysbiosis in patients with COVID-19}

217 Alterations in the microbial diversity prompted us to determine microbial dysbiosis index (DI) 218 (variations in the microbial community) across the three groups (C, IA, and IS). We performed 219 PCoA using the Bray Curtis distance matrix and found that NP microbiota was significantly 220 altered $(\mathrm{p}=0.001)$ with $61 \%$ variation in distances explained $\left(\mathrm{R}^{2}=0.6136\right)$, as assessed using 221 the ADONIS test. Next, we calculated the Euclidean distance from the centroid for samples 222 from control $($ median $=0.3404)$, asymptomatic $($ median $=0.1881)$ and symptomatic $($ median $223=0.1511)$ individuals and calculated the DI (Supplementary figure 1B). The overall observed 224 DI was significant (Kruskal-Wallis test, $\mathrm{p}=1.317 \mathrm{E}-07$ ) across all the groups. Pairwise 225 comparison showed significant dysbiosis between control vs. symptomatic $(\mathrm{p}=5.6 \mathrm{E}-09)$ and 226 control vs. asymptomatic $(\mathrm{p}=1.1 \mathrm{E}-09)$ groups; however, dysbiosis between asymptomatic 227 and symptomatic $(\mathrm{p}=0.016)$ pair was also significant, although the value was moderately low 
compared to those of the other comparisons (Figure $2 \mathrm{H}$ ). We also observed highly significant dysbiosis $(\mathrm{p}=2.2 \mathrm{E}-12)$ between the control and infected groups (Supplemental Figure 1A, 1C). This showed that compared to control subjects, the NP microbial community was severely altered in both symptomatic and asymptomatic COVID-19 patients.

Distinct microbial composition and abundance at phylum and family levels in patients altered in COVID-19 patients. Next, we aimed to identify the microbial communities that were altered at different taxonomic levels in three sample groups. We found 795 differential OTUs, out of which 12 phyla, 65 orders, 126 families, and 240 genera were present in all three groups (C, IA, and IS) (Supplemental Table 1). The 12 phyla and their significance is shown in Table 2. The most significant phyla in the SARS-CoV-2-infected groups (symptomatic and asymptomatic) were Actinobacteria $(p=9.96 \mathrm{E}-07)$, Firmicutes $(\mathrm{p}=4.65 \mathrm{E}-02)$, and Proteobacteria ( $\mathrm{p}=9.61 \mathrm{E}-07)$, including 8 other phyla assessed using the Kruskal-Wallis test. In contrast, Bacteroidetes $(\mathrm{p}=1.48 \mathrm{E}-06)$ and Proteobacteria $(\mathrm{p}=6.56 \mathrm{E}-07)$ were highly abundant in the control group (non-infected) (Supplemental Figure 2A). Furthermore, we analyzed the relative abundance of the top 30 families and found enrichment of Mycobacteriaceae, Propionibacteriaceae, and Streptomycetaceae (Supplemental Figure 2B). These families contain opportunistic pathogens, which were found in both symptomatic and asymptomatic COVID-19 patients, while they were absent in the healthy controls. The top families and their significance is shown in Table 3. 
251 To further our understanding regarding the 795 differentially abundant OTUs, we used the 252 NMDS approach at phylum, order, family, and genus levels for C, IA, and IS sample groups 253 using the Bray-Curtis distance matrix. Analysis of statistical significance using analysis of 254 similarities (ANOSIM) for phylum $(R=0.262, p=1.7 \mathrm{E}-03)$, order $(R=0.322, p=3 \mathrm{E}-04)$, 255 family $(R=0.3461, p=3 E-04)$, and genus $(R=0.3507, p=3 E-04)$ showed gradual increase 256 in R-value for genus. This indicated that as we go lower in taxonomic classification, the 257 variance in the OTUs provides better sample segregation. The differential OTUs present at the 258 genus level in three sample groups showed significant level of dissimilarity $(p=3 E-04)$ with $259 \mathrm{R}=0.3507$ and clear sample segregation (Figure 3A). We used sample correlation (Spearman 260 matrix) to further validate the NMDS findings and identify the NP OTU differences between 261 C, IA, and IS sample groups (Supplemental Figure 3A-D). To further reconcile the distinct 262 sample segregation at higher to lower taxonomic level based on OTU abundances, we plotted 263 density histogram of correlation coefficient values (obtained in sample correlation). The mean 264 and median value of each density plot revealed lack of difference between the C, IA and IS 265 groups at the phylum level and subtle differences were observed at the order and family levels. 266 However, at the genus level, we found comprehensible differences between $\mathrm{C}(\mathrm{mean}=7.95 \mathrm{E}$ $26701 ;$ median $=6.39 \mathrm{E}-01)$, IA $($ mean $=5.65 \mathrm{E}-01 ;$ median $=8.33 \mathrm{E}-01)$, and IS $($ mean $=6.51 \mathrm{E}-$ 26801 ; median $=7.01 \mathrm{E}-01)($ Table 4$)($ Figure $3 \mathrm{~B})$. To evaluate the statistical significance of 269 densities based on sample segregation, we calculated cumulative distribution distance (D) and 270 significance between C, IA, and IS groups using the KS test for each taxonomic rank (Table 271 5). Based on the ' $D$ ' value, the samples were well distributed in $C$ vs. IA ( $D=5.94 E-01$; p272 value $<2.2 \mathrm{E}-16), \mathrm{C}$ vs. IS $(\mathrm{D}=5.06 \mathrm{E}-01 ; \mathrm{p}=3.308 \mathrm{E}-14)$, and IA vs. IS $(\mathrm{D}=2.28 \mathrm{E}-01 ; \mathrm{p}=$ $273<2.2 \mathrm{E}-16)$ at the genus level. The differences in overall sample distribution were more highly 
274 enriched at the genus level than between these three groups. Although the 'D' value between

275 IA and IS groups was less, their distribution pattern differed significantly. Based on the above

276 observations, we considered the genus level OTUs $(n=240)$ for downstream analysis.

\section{Cluster-specific OTUs at genus level revealed unique sample-specific OTUs}

278 To gain insights regarding how the bacterial genera were segmented among three groups of 279 samples, we performed genus level OTU correlation $(n=240)$ and calculated the correlation 280 coefficient (Spearman correlation), followed by unsupervised hierarchical clustering (Figure 281 3C). We identified five distinct clusters, $C 1(n=23), C 2(n=109), C 3(n=59), C 4(n=33)$

282 and C5 $(\mathrm{n}=16)$, with variable number of OTUs (Supplemental Table 2). The heat map 283 corresponding to each cluster is shown in Supplemental Figure 4. The relative abundance 284 density maps of cluster-wise OTUs were constructed, which distinguished OTUs that were 285 enriched in IA/IS (C1, C3, C4, and C5) and in the control samples (C2) (Figure 3D). Some of 286 the enriched cluster-specific OTUs in IA/IS are Mycobacterium (1763), Mycolicibacterium 287 (1766), Mycobacteroides (1774), Halothiobacillus (927), Flavobacterium (986), 288 Bifidobacterium (1695), Streptomyces (1884), Rothia (2047), and Mycoplasma (2100). C2, a 289 control-specific cluster, contained OTUs such as Thermomicrobium (500), Kingella (502), 290 Enterobacter (547), Bacteroides (821), and Prevotella (840). Thus, this analysis shows the 291 distinction in genus-specific OTUs for both SARS-CoV-2-infected and control subjects. Next, 292 we performed CCA on each of the clusters (C1 to C5) to eliminate sample heterogeneity and 293 enhance the stringency of our analysis pipeline (Figure 3E). We considered the first two 294 components of CCA that explained cumulative variance for the clusters. Cluster C4 explained 295 the highest cumulative variance of $94.8 \%$, while cluster C3 showed the lowest variance of 
12.9\% (Figure 3F, Supplemental Figure 5A-E). CCA showed efficient sample clustering, 297 which is reminiscent of the density plot (Figure 3D-E).

\section{LefSe analysis identified unique OTUs at genus level in patients with COVID-19}

299 The CCA analysis prompted us to select clusters with maximum variance explained. 300 Therefore, we considered all clusters with $\geq 30 \%$ variance, which includes all the clusters, 301 except C3, for LDA. OTUs $(n=181)$ were extracted from clusters $(C 1, C 2, C 4, C 5)$ and plotted 302 in a heat map with their abundance (Figure 4A). Different genera could be clearly 303 distinguished between C, IA, and IS sample groups. Next, we performed LefSe to distinguish 304 the most significant microbiomes from C, IA, and IS groups. In a one-against-all comparison 305 (C with IA and IS), we obtained 40 genera in the control group, 34 genera in the symptomatic 306 group, and 4 genera in the asymptomatic group (LDA score $[\log 10]>3.6$ ). The genera 307 obtained from one-against-all are highlighted in the heatmap (Figure 4A and Supplemental 308 Figure 6A). Relative abundance of each OTU obtained from C, IA, and IS groups are shown 309 in stack plots, with clear segregation in OTUs for individual samples (Supplemental Figure 310 6B-D). The DI calculated from these genera showed high dysbiosis between control and 311 SARS-CoV-2-infected patients (Supplemental Figure 6E-F). We further increased the LefSe stringency by using all-against-all (each sample group compared with each other) comparisons 313 and constructed a cladogram and a bar plot (Figure 4B-C). All the genera obtained from LefSe 314 (one-against-all and all-against-all) with their LDA scores and comparison are listed in 315 Supplemental Table 3 and Table 6. We obtained 12 significantly enriched genera of 316 Gallibacterium, Orientia, Acidocella, and Citrobacter in control samples (LDA score $317[\log 10]>2.0)$, Mycoplasma, Streptosporangium, Mycobacterium, Mycolicibacterium, 318 Mycolicibacillus, and Mycobacteroides in symptomatic samples, and Oerskovia and 
Cellulosimicrobium in asymptomatic samples (Figure 4C). The histogram showing the relative abundance of the 12 genera for C, IA, and IS sample groups clearly distinguished each sample type (Figure 4D). Finally, we used weighted correlation network analysis to construct a network (Spearman correlation) with 12 genera identified using the LDA analysis. The network creates two distinct modules, one for control groups and another for both symptomatic and asymptomatic groups. We obtained strong association within the genera of C, IA, and IS sample groups (Table 7). However, the correlation between C vs. IA was extremely weak and correlation was not obtained for C vs. IS groups. The network analysis suggested that the NP microbiota of the control group was clearly distinct from that of the asymptomatic and symptomatic groups. The DI of the 12 genera showed the highest significance between $\mathrm{C}$ vs. IS $(\mathrm{p}=4.7 \mathrm{E}-05)$, while significant dysbiosis was not observed between IA and IS groups (Figure 4F). Overall, our analysis confirmed the significance of the genera identified and their associations with symptomatic and asymptomatic COVID-19 patients.

333 After successful identification of 12 genera based on LDA score from all-against-all comparisons, we investigated different species-specific OTUs associated with each of the 12 genus and identified 54 species represented in the heatmap (Figure 6A). The differential abundance of several known opportunistic pathogens such as Mycobacterium tuberculosis, Mycobacterium avium Mycoplasma pneumoniae, Mycobacterium leprae, and Mycobacterium kansasii were significantly high in the SARS-CoV2 infected patients (Figure 6A-D). The pairwise comparison and significance of the relative abundance of 12 previously reported pathogens are shown in Figure 6B-D and Table 8. We found that all 12 pathogenic species, including Mycobacterium tuberculosis, Mycobacterium avium, Mycobacterium intracellulare 
(MAC), Mycoplasma pneumoniae, Mycobacterium leprae, Mycobacterium Kansasii, Mycobacterium simiae, Mycobacterium xenopi, Mycobacterium gordonae, Mycobacterium asiaticum, Mycobacterium szulgai, and Mycobacterium ulcerans were more abundant in symptomatic patients than in asymptomatic patients (Figure 6D). Although abundance of these pathogens was obtained in both IA and IS subjects compared to that in the controls, the overall

347 enrichment in the IS subjects were higher, indicating their possible role in secondary 348 infections.

\section{Distinct correlation of OTUs with clinical symptoms in patients with COVID-19}

To evaluate the accuracy of LDA classification that identified eight bacterial genera in the IA and IS sample group, we tested the ROC (receiver operating characteristics)-AUC (area under

352 the curve) score. We obtained a value of 0.8 with $95 \%$ confidence interval for true positive 353 classification, showing $80 \%$ sensitivity and specificity of data obtained from LDA analysis 354 (Figure 5A). Next, we used the Spearman correlation matrix to identify the association of 355 symptoms with the genera. Interestingly, chest pain showed high positive correlation with the 356 presence of Mycoplasma, Mycobacterium, Mycolicibacterium, Mycolicibacillus, and 357 Mycobacteroides, which were related to the IS group, and weak correlation with Oerskovia and Cellulosimicrobium, which were associated with the IA group. Mycoplasma, however, 359 showed strong correlation with both chest pain (0.4446) and fever (0.4214) (Figure 5B). ROC360 AUC analysis for chest pain and fever yielded 0.90 and 0.79 scores, respectively, with eight 361 bacterial genera (Figure 5C-D). At the species level, fever correlated strongly with most 362 Mycobacterium spp. and Mycoplasma spp., which were significantly abundant in symptomatic 363 COVID-19 patients (Figure 6E). In short, we established the association of pathogenic 364 microbes with COVID-19 and showed susceptibility to alterations in the NP microbiome in 
case of co-infections in SARS-CoV-2. We also identified the compositional difference in NP

366 microbiota between symptomatic and asymptomatic group.

\section{Discussion}

368 Although evidence regarding changes in NP microbiota in COVID-19 patients is available, 369 data regarding association of opportunistic pathogens with disease symptoms, indicative of 370 co-infections, are limiting; in addition, evidence regarding the presence of asymptomatic- and 371 symptomatic patient-specific bacterial communities is still awaited. Previous studies have 372 revealed the association of disease-specific opportunistic pathogens in respiratory diseases 373 such as chronic rhinosinusitis, asthma, pneumonia, and cystic fibrosis (7-10). Therefore, we 374 aimed to identify the NP microbial alterations, opportunistic pathogens, and their association 375 with COVID-19-positive asymptomatic and symptomatic subjects. In this study, we were able 376 to distinguish the microbial community associated with the control, symptomatic, and 377 asymptomatic COVID-19 patients.

378 Scientists worldwide are attempting to understand the pathophysiology of SARS-CoV-2 379 infection and the associated co-infections/secondary infections that may arise due to the 380 alterations in the host microbiome. As SARS-CoV-2 infection initiates in the upper respiratory 381 tract, we investigated the alterations in the NP microbiota of COVID-19 patients. We 382 amplified the 16S rRNA gene of variable regions (V1-V9) and performed long-read 383 sequencing using Oxford Nanopore technology. Subsequently, we have used multiple 384 bioinformatics approaches to cross-validate our data sets at various levels and identify the 385 most significant bacterial population in the NP microbiome of COVID-19 patients. We found 386 significant changes in microbial abundance/diversity and DI in SARS-CoV-2-infected patients 387 compared to those of the control. The IA and IS groups also showed overall significant 
alterations in microbiota composition. We found abundance of opportunistic pathogens such as Mycoplasma and Mycobacterium in symptomatic patients, which correlated strongly with patient symptoms such as chest pain and fever. Insights into species level revealed the 391 abundance of 12 different bacterial species belonging to the genus Mycoplasma and 392 Mycobacterium in the SARS-CoV-2-infected patients. To the best of our knowledge, this is 393 the first comprehensive and systematic cohort-based study to report the abundance of 394 opportunistic pathogens Mycoplasma and Mycobacterium using long read sequencing of $16 \mathrm{~S}$ 395 rRNA variable regions in patients with SARS-CoV-2 infection.

396 Respiratory infections alter the NP microbiota, reducing microbial diversity, and promotes the 397 growth of opportunistic pathogens (26). Proteobacteria, Firmicutes, and Actinobacteria are 398 present in all NP samples at the phylum level, however, the abundance of Firmicutes and 399 Actinobacteria was significantly higher in both symptomatic and asymptomatic COVID-19 400 patients in our study. Our results are in partial agreement with those of Ventero et al., who 401 found the abundance of Firmicutes, Bacteroidota, Proteobacteria, and Actinobacteria in the 402 NP samples of COVID-19 patients (13). Maio et al. and Braun et al. did not find any 403 significant alterations in NP microbial composition $(12,27)$. Other studies have shown the 404 prevalence of opportunistic pathogens such as Staphylococcus, Anelloviridae, Pseudomonas, 405 Haemophilus, Stenotrophomonas, Redondoviridae, and Pseudomonas aeruginosa in COVID40619 patients $(11,13,15-18)$. Compared to earlier reports, our study also revealed overall 407 changes in the composition of the NP microbial community, reduction in bacterial diversity 408 and the presence of opportunistic pathogens.

409 Most of the NP microbial studies involve amplification of the shorter 16S rRNA variable 410 region using the Illumina platform, which is more accurate, but is limited by taxonomic 
411 resolution owing to sequencing of shorter reads and specific variable region (28). The 412 taxonomic resolution can be improved to genus, species, and even at the strain level by 413 sequencing the V1 $\square \mathrm{V} 9(\sim 1600 \mathrm{bp})$ variable regions of the $16 \mathrm{~S}$ rRNA gene $(28,29)$. In this 414 study, we have successfully used the Oxford Nanopore long read sequencing platform and 415 sequenced $\mathrm{V} 1$ to $\mathrm{V} 9(\sim 1.6 \mathrm{~kb})$ of the $16 \mathrm{~S}$ rDNA variable regions to obtain taxonomic 416 resolution to genus and species level. This has provided us immense advantage in determining 417 the abundance of opportunistic pathogens in the NP of the COVID-19 patients. Until now, 418 only Mostafa et al. has used metagenomics for COVID-19 NP samples using Oxford Nanopore 419 technology. They have sequenced both RNA and DNA from the NP samples without any PCR 420 amplification. They not only identified the SARS-CoV-2 virus in the samples, but also 421 potential pathogens that may lead to co-infections (18). This is the first comprehensive study 422 on high throughput analysis of the NP microbiome in COVID-19 patients from an Odisha 423 cohort and the second in India after Gupta et al., who used the Illumina platform for 16S 424 amplicon sequencing and found enrichment of several opportunistic pathogens (17). 425 Interestingly, Mycoplasma, Mycolicocibacterium, and Mycobacterium were not present in 426 their list of opportunistic pathogens. This could be due to the short reads sequenced, and 427 analysis pipeline and/or geographical region-specific differences. Nevertheless, the 428 identification of opportunistic pathogens and increase in their abundance in COVID-19 429 patients is one of the important aspects of this study.

430 SARS-CoV-2 infections elicits strong immune responses in the host, leading to cytokine 431 storm, which also affects normal cells. The administration of immune suppressants 432 compromise patient's immunity and render them prone to co-infections with other 433 opportunistic pathogens, worsening the pathophysiology of the COVID-19 patients (19, 20). 
434 Our comprehensive bioinformatics analysis with sample-sample and OTU-OTU correlation 435 analysis distinguished COVID-19-infected and control specific OTUs at the genus level. 436 Furthermore, LDA and network analysis identified significantly high abundance of 437 Mycobacterium and Mycoplasma in symptomatic patients, which correlated well with the 438 occurrence of fever and chest pain. Significantly high relative abundance of members of 439 family Mycobacteriaceae in the symptomatic COVID-19 group indicates the presence of both 440 pathogenic and non-pathogenic bacteria. Members of the Mycobacterium genera are well 441 associated with several pulmonary diseases; for example, Mycobacterium tuberculosis is 442 responsible for tuberculosis in humans and is associated with pulmonary infection (30), while 443 Mycobacterium avium is highly associated with lung disease (31). Interestingly, several case 444 reports from China, Mexico, India, and Singapore have identified Mycobacterium tuberculosis 445 as co-infections in COVID-19 patients and were treated with drugs to combat tuberculosis 446 (32-36). Two meta-analysis studies clearly showed Mycobacterium tuberculosis co-infection 447 in patients with COVID-19, which renders the patients vulnerable to severe COVID-related 448 complications and death (37-41). As per WHO report, around 10 million people were affected 449 by tuberculosis in 2020 and eight countries accounted for $2 / 3^{\text {rd }}$ of patients with tuberculosis 450 infection. Reports show susceptibility for tuberculosis and activation of latent tuberculosis 451 during COVID-19 infections (36-38). Therefore, this study is particularly important for 452 countries such as China, India, Indonesia, South Africa, Bangladesh, Pakistan, Philippines, 453 and Nigeria, where tuberculosis is endemic and possibly aggravates the COVID-19 symptoms 454 and severity. Studies have revealed that patients suffering from tuberculosis are more 455 susceptible for SARS-CoV-2 infection, faster disease progression, and complications 456 associated with COVID-19 disease $(36,38)$. 
457 Mycoplasma pneumoniae, a member of genus Mycoplasma, is responsible for pneumonia and 458 other respiratory infections in humans $(42,43)$. Co-infections and/or secondary infections by 459 Mycoplasma pneumoniae is not uncommon in COVID-19 patients. Several case reports from 460 different parts of the world have shown that Mycoplasma pneumoniae co-infections in 461 COVID-19 patients result in disease severity (44-51). Patients with atypical respiratory 462 pathogens such as Mycoplasma pneumoniae or Chlamydia pneumoniae co-infections have 463 high propensity of developing acute respiratory distress syndrome (ARDS) (45). Both 464 COVID-19 pneumonia and pulmonary pneumonia elicit fever, cough, and shortness of breath, 465 and present similar diagnostic features in chest X-ray and HRCT, because of which they 466 escape logical diagnosis by clinicians. Serological tests can reveal the presence of 467 Mycoplasma pneumoniae in COVID-19 patients. The results of our study and those of other 468 reports have proved the association of opportunistic pathogens with alterations in the diversity 469 of the microbial communities in symptomatic and asymptomatic COVID-19 patients. This 470 study establishes a new set of opportunistic pathogens in the context of the NP microbiome in 471 COVID-19-infected patients. Moreover, this study clearly distinguishes between the NP 472 microbial composition of symptomatic and asymptomatic groups using LefSe with AUC-ROC 473 validation. Thus, we believe that SARS-CoV-2 virulence may promote the growth of 474 opportunistic pathogens and may lead to co-infection or secondary infection in COVID-19 475 patients. Our study also highlights the importance of incorporating routine screening for 476 common opportunistic pathogens in COVID-19 patients in public health policies. Timely 477 identification and medications may regulate the occurrence of co-infections and may reduce 478 disease burden, along with faster recovery from SARS-CoV-2 infections. 
479 Our study has certain limitations. The subject size is limited and a larger cohort would have 480 strengthened our findings. The clinical manifestations are limited, and therefore, we have 481 attempted not to over interpret our data. Future studies should include NP samples from 482 vaccinated, asymptomatic, and hospitalized COVID-19 patients, the detailed associated 483 pathophysiology of whom are known, to understand the role of opportunistic pathogens in 484 COVID-19 disease progression and severity. Furthermore, blood biochemistry and serum 485 metabolite studies will boost conclusions regarding the functional aspects of the NP 486 microbiome.

$488 \quad$ Conclusions

489 In this study, we report the abundance of Mycobacteria and Mycoplasma opportunistic 490 pathogens in the nasopharynx of non-hospitalized COVID-19 patients. Using long-read 491 sequencing of the 16S rRNA variable regions V1-V9, we extended our analysis to species 492 level and found enrichment of several Mycobacterium spp. and Mycoplasma pneumoniae in 493 both asymptomatic and symptomatic patients with COVID-19. However, their abundance was 494 significantly higher in the symptomatic COVID-19 patients, with strong association with chest 495 pain and fever. Our study adds to the growing list of opportunistic pathogens in patients with 496 COVID-19 and indicates the possibility of co-infections during disease progression. Hence, 497 we believe that it is important to incorporate routine screening for common opportunistic 498 pathogens in SARS-CoV-2-infected patients and modulate treatment modalities to prevent co499 infections and secondary infections. 
The 16S rRNA gene sequencing data has been deposited in NCBI repository with the

503 Bioproject ID for PRJNA774098 (http://www.ncbi.nlm.nih.gov/bioproject/774098).

504

$505 \quad$ References

506 1. He Y, Wang J, Li F, Shi Y. Main Clinical Features of COVID-19 and Potential Prognostic

507 and Therapeutic Value of the Microbiota in SARS-CoV-2 Infections. Front Microbiol. $508 \quad 2020 ; 11: 1302$.

509 2. Khatiwada S, Subedi A. Lung microbiome and coronavirus disease 2019 (COVID-19):

$510 \quad$ Possible link and implications. Hum Microb J. 2020;17:100073.

511 3. Zou X, Chen K, Zou J, Han P, Hao J, Han Z. Single-cell RNA-seq data analysis on the 512 receptor ACE2 expression reveals the potential risk of different human organs vulnerable 513 to 2019-nCoV infection. Front Med. 2020;14(2):185-92.

514 4. Hou YJ, Okuda K, Edwards CE, Martinez DR, Asakura T, Dinnon KH, 3rd, et al. SARS515 CoV-2 Reverse Genetics Reveals a Variable Infection Gradient in the Respiratory Tract. Cell. 2020;182(2):429-46 e14.

517 5. Mlcochova P, Kemp SA, Dhar MS, Papa G, Meng B, Ferreira I, et al. SARS-CoV-2 518 B.1.617.2 Delta variant replication and immune evasion. Nature. 2021.

519 6. Belkaid Y, Harrison OJ. Homeostatic Immunity and the Microbiota. Immunity. $520 \quad 2017 ; 46(4): 562-76$.

521 7. Fazlollahi M, Lee TD, Andrade J, Oguntuyo K, Chun Y, Grishina G, et al. The nasal 522 microbiome in asthma. J Allergy Clin Immunol. 2018;142(3):834-43 e2. 
523 8. de Steenhuijsen Piters WA, Sanders EA, Bogaert D. The role of the local microbial ecosystem in respiratory health and disease. Philos Trans R Soc Lond B Biol Sci. 2015;370(1675).

526 9. Kumpitsch C, Koskinen K, Schopf V, Moissl-Eichinger C. The microbiome of the upper 527 respiratory tract in health and disease. BMC Biol. 2019;17(1):87.

528 10. Yildiz S, Mazel-Sanchez B, Kandasamy M, Manicassamy B, Schmolke M. Influenza A 529

11. Engen PA, Naqib A, Jennings C, Green SJ, Landay A, Keshavarzian A, et al. Nasopharyngeal Microbiota in SARS-CoV-2 Positive and Negative Patients. Biol Proced Online. 2021;23(1):10.

534 12. De Maio F, Posteraro B, Ponziani FR, Cattani P, Gasbarrini A, Sanguinetti M. Online. 2020;22:18.

13. Ventero MP, Cuadrat RRC, Vidal I, Andrade BGN, Molina-Pardines C, Haro-Moreno JM, et al. Nasopharyngeal Microbial Communities of Patients Infected With SARS-CoV-2 That Developed COVID-19. Front Microbiol. 2021;12:637430.

14. Feehan AK, Rose R, Nolan DJ, Spitz AM, Graubics K, Colwell RR, et al. Nasopharyngeal Microbiome Community Composition and Structure Is Associated with Severity of COVID-19 Disease and Breathing Treatment. Applied Microbiology. 2021;1(2):177-88.

15. Rhoades NS, Pinski AN, Monsibais AN, Jankeel A, Doratt BM, Cinco IR, et al. Acute SARS-CoV-2 infection is associated with an increased abundance of bacterial pathogens, including Pseudomonas aeruginosa in the nose. Cell Rep. 2021;36(9):109637. 
546 16. Nardelli C, Gentile I, Setaro M, Di Domenico C, Pinchera B, Buonomo AR, et al. 547 Nasopharyngeal Microbiome Signature in COVID-19 Positive Patients: Can We 548 Definitively Get a Role to Fusobacterium periodonticum? Front Cell Infect Microbiol. $549 \quad 2021 ; 11: 625581$.

550 17. Gupta A, Karyakarte R, Joshi S, Das R, Jani K, Shouche Y, et al. Nasopharyngeal 551 microbiome reveals the prevalence of opportunistic pathogens in SARS-CoV-2 infected 552 individuals and their association with host types. Microbes Infect. 2021:104880.

553 18. Mostafa HH, Fissel JA, Fanelli B, Bergman Y, Gniazdowski V, Dadlani M, et al. 554 Metagenomic Next-Generation Sequencing of Nasopharyngeal Specimens Collected from 555 Confirmed and Suspect COVID-19 Patients. mBio. 2020;11(6):1-13.

556 19. Abdoli A, Falahi S, Kenarkoohi A. COVID-19-associated opportunistic infections: a 557 snapshot on the current reports. Clin Exp Med. 2021.

558 20. Fishman JA. Opportunistic infections--coming to the limits of immunosuppression? Cold $559 \quad$ Spring Harb Perspect Med. 2013;3(10):a015669.

560 21. Wood DE, Lu J, Langmead B. Improved metagenomic analysis with Kraken 2. Genome $561 \quad$ Biol. 2019;20(1):257.

562 22. Varet H, Brillet-Gueguen L, Coppee JY, Dillies MA. SARTools: A DESeq2- and EdgeR$563 \quad$ Based R Pipeline for Comprehensive Differential Analysis of RNA-Seq Data. PLoS One. 564 2016;11(6):e0157022.

565 23. Lloyd-Price J, Arze C, Ananthakrishnan AN, Schirmer M, Avila-Pacheco J, Poon TW, et 566 al. Multi-omics of the gut microbial ecosystem in inflammatory bowel diseases. Nature. 567 2019;569(7758):655-62. 
568 24. Langfelder P, Horvath S. WGCNA: an R package for weighted correlation network $569 \quad$ analysis. BMC Bioinformatics. 2008;9:559.

570 25. Bastian M, Heymann S, Jacomy M. Gephi: An Open Source Software for Exploring and 571 Manipulating Networks. Proceedings of the International AAAI Conference on Web and $572 \quad$ Social Media. 2009;3(1):361-2.

573 26. Santacroce L, Charitos IA, Ballini A, Inchingolo F, Luperto P, De Nitto E, et al. The 574 Human Respiratory System and its Microbiome at a Glimpse. Biology (Basel). 2020;9(10).

575 27. Braun T, Halevi S, Hadar R, Efroni G, Glick Saar E, Keller N, et al. SARS-CoV-2 does 576 not have a strong effect on the nasopharyngeal microbial composition. Sci Rep. $577 \quad 2021 ; 11(1): 8922$.

578 28. Johnson JS, Spakowicz DJ, Hong BY, Petersen LM, Demkowicz P, Chen L, et al. 579 Evaluation of 16S rRNA gene sequencing for species and strain-level microbiome analysis. $580 \quad$ Nat Commun. 2019;10(1):5029.

581 29. Kaul D, Rathnasinghe R, Ferres M, Tan GS, Barrera A, Pickett BE, et al. Microbiome 582 disturbance and resilience dynamics of the upper respiratory tract during influenza A virus $583 \quad$ infection. Nat Commun. 2020;11(1):2537.

584 30. Peto HM, Pratt RH, Harrington TA, LoBue PA, Armstrong LR. Epidemiology of 585 extrapulmonary tuberculosis in the United States, 1993-2006. Clin Infect Dis. 586 2009;49(9):1350-7.

587 31. Hwang JA, Kim S, Jo KW, Shim TS. Natural history of Mycobacterium avium complex 588 lung disease in untreated patients with stable course. Eur Respir J. 2017;49(3):1600537. 
589 32. Yao Z, Chen J, Wang Q, Liu W, Zhang Q, Nan J, et al. Three Patients with COVID-19 and 590 Pulmonary Tuberculosis, Wuhan, China, January-February 2020. Emerg Infect Dis. 2020;26(11):2755-8.

592 33. Sreenath K, Batra P, Vinayaraj EV, Bhatia R, SaiKiran K, Singh V, et al. Coinfections with 593 Other Respiratory Pathogens among Patients with COVID-19. Microbiol Spectr. 2021;9(1):e0016321.

595 34. Tham SM, Lim WY, Lee CK, Loh J, Premkumar A, Yan B, et al. Four Patients with COVID-19 and Tuberculosis, Singapore, April-May 2020. Emerg Infect Dis.

598 35. Visca D, Ong CWM, Tiberi S, Centis R, D'Ambrosio L, Chen B, et al. Tuberculosis and 599 COVID-19 interaction: A review of biological, clinical and public health effects. 600 Pulmonology. 2021;27(2):151-65.

601 36. Gerstein S, Khatri A, Roth N, Wallach F. Coronavirus disease 2019 and extra-pulmonary 602 tuberculosis co-infection - A case report and review of literature. J Clin Tuberc Other Mycobact Dis. 2021;22:100213.

604 37. Gao Y, Liu M, Chen Y, Shi S, Geng J, Tian J. Association between tuberculosis and 605 COVID-19 severity and mortality: A rapid systematic review and meta-analysis. J Med 606 Virol. 2021;93(1):194-6.

607 38. Song WM, Zhao JY, Zhang QY, Liu SQ, Zhu XH, An QQ, et al. COVID-19 and 608 Tuberculosis Coinfection: An Overview of Case Reports/Case Series and Meta-Analysis. 609 Front Med (Lausanne). 2021;8:657006. 
610

611

612

613

614

615

616

617

618

619

620

621

622

623

624

625

626

627

628

629

630

631

632

39. Martinez Orozco JA, Sanchez Tinajero A, Becerril Vargas E, Delgado Cueva AI, Resendiz Escobar H, Vazquez Alcocer E, et al. COVID-19 and Tuberculosis Coinfection in a 51Year-Old Taxi Driver in Mexico City. Am J Case Rep. 2020;21:e927628.

40. He G, Wu J, Shi J, Dai J, Gamber M, Jiang X, et al. COVID-19 in tuberculosis patients: A report of three cases. J Med Virol. 2020;92(10):1802-6.

41. Tadolini M, Codecasa LR, Garcia-Garcia JM, Blanc FX, Borisov S, Alffenaar JW, et al. Active tuberculosis, sequelae and COVID-19 co-infection: first cohort of 49 cases. Eur Respir J. 2020;56(1).

42. Beeton ML, Zhang XS, Uldum SA, Bebear C, Dumke R, Gullsby K, et al. Mycoplasma pneumoniae infections, 11 countries in Europe and Israel, 2011 to 2016. Euro Surveill. $2020 ; 25(2)$.

43. Foy HM. Infections caused by Mycoplasma pneumoniae and possible carrier state in different populations of patients. Clin Infect Dis. 1993;17 Suppl 1:S37-46.

44. Amin D, McKitish K, Shah PS. Association of mortality and recent Mycoplasma pneumoniae infection in COVID-19 patients. J Med Virol. 2021;93(2):1180-3.

45. Chaudhry R, Sreenath K, Batra P, Vinayaraj EV, Rathor N, Saikiran K, et al. Atypical bacterial co-infections among patients with COVID-19: A study from India. J Med Virol. 2022;94(1):303-9.

46. Chen S, Zhu Q, Xiao Y, Wu C, Jiang Z, Liu L, et al. Clinical and etiological analysis of co-infections and secondary infections in COVID-19 patients: An observational study. Clin Respir J. 2021;15(7):815-25.

47. Tang ML, Li YQ, Chen X, Lin H, Jiang ZC, Gu DL, et al. Co-Infection with Common Respiratory Pathogens and SARS-CoV-2 in Patients with COVID-19 Pneumonia and 

from a Single Center in China. Med Sci Monit. 2021;27:e929783.

635 48. Huang AC, Huang CG, Yang CT, Hu HC. Concomitant infection with COVID-19 and 636 Mycoplasma pneumoniae. Biomed J. 2020;43(5):458-61.

637 49. Chaudhry R, Sreenath K, Vinayaraj EV, Sahoo B, Vishnu Narayanan MR, Kiran K, et al. 638 639 Mycoplasma pneumoniae co-infection with SARS-CoV-2: A case report. Access Microbiol. 2021;3(3):000212.

640 50. Gayam V, Konala VM, Naramala S, Garlapati PR, Merghani MA, Regmi N, et al. 641 Presenting characteristics, comorbidities, and outcomes of patients coinfected with 642 COVID-19 and Mycoplasma pneumoniae in the USA. J Med Virol. 2020;92(10):2181-7.

643 51. Choubey A, Sagar D, Cawley P, Miller K. Retrospective review analysis of COVID-19 644 patients co-infected with Mycoplasma pneumoniae. Lung India. 645 646 52. Operario DJ, Pholwat S, Koeppel AF, Prorock A, Bao Y, Sol-Church K, et al.

53. Martins AB, Matos ED, Lemos AC. Infection with the Mycobacterium avium complex in 650 651 patients without predisposing conditions: a case report and literature review. Braz J Infect Dis. 2005;9(2):173-9.

652 54. Shah NM, Davidson JA, Anderson LF, Lalor MK, Kim J, Thomas HL, et al. Pulmonary 653 Mycobacterium avium-intracellulare is the main driver of the rise in non-tuberculous mycobacteria incidence in England, Wales and Northern Ireland, 2007-2012. BMC Infect 655 Dis. 2016;16:195. 
656 55. Daley CL, Winthrop KL. Mycobacterium avium Complex: Addressing Gaps in Diagnosis and Management. J Infect Dis. 2020;222(Suppl 4):S199-S211.

658 56. Yang JJ, Mohallem DF, Cardoso TA, Lima Junior CL, Tebcherani AJ, Vidigal Mdo R. 659 Case for diagnosis. An Bras Dermatol. 2014;89(5):837-8.

660 57. Cerqueira S, Deps PD, Cunha DV, Bezerra NVF, Barroso DH, Pinheiro ABS, et al. The 661

58. Zhang M, Feng M, He JQ. Disseminated Mycobacterium kansasii infection with cutaneous 665

59. Johnston JC, Chiang L, Elwood K. Mycobacterium kansasii. Microbiol Spectr. 2017;5(1). 667

60. van Ingen J, Boeree MJ, Dekhuijzen PN, van Soolingen D. Clinical relevance of 668 669 Mycobacterium simiae in pulmonary samples. Eur Respir J. 2008;31(1):106-9.

61. Hamieh A, Tayyar R, Tabaja H, S ELZ, Bou Khalil P, Kara N, et al. Emergence of Mycobacterium simiae: A retrospective study from a tertiary care center in Lebanon. PLoS One. 2018;13(4):e0195390.

62. Okano Y, Shinohara T, Imanishi S, Takahashi N, Naito N, Taoka T, et al. Miliary pulmonary nodules due to Mycobacterium xenopi in a steroid-induced immunocompromised patient successfully treated with chemotherapy: a case report. BMC Pulm Med. 2016;16(1):92.

63. Gochi M, Kaneko Y, Seki A, Saitou Z, Samejima T, Seki Y, et al. [Mycobacterium xenopi lung infection in a patient with multiple lung cysts that responded well to chemotherapy: a case report]. Kekkaku. 2012;87(11):733-6. 
679 64. Research Committee of the British Thoracic S. First randomised trial of treatments for 680 pulmonary disease caused by $\mathrm{M}$ avium intracellulare, $\mathrm{M}$ malmoense, and $\mathrm{M}$ xenopi in HIV negative patients: rifampicin, ethambutol and isoniazid versus rifampicin and ethambutol. Thorax. 2001;56(3):167-72.

683 65. Youssef D, Shams WE, Elshenawy Y, El-Abbassi A, Moorman JP. Pulmonary infection 684 685 686 687

68. Arttawejkul P, Kongpolprom N. A case of pulmonary infection caused by Mycobacterium asiaticum: Difficulties on diagnostic and therapeutic approaches. Respir Med Case Rep.

69. van Ingen J, Boeree M, Janssen M, Ullmann E, de Lange W, de Haas P, et al. Pulmonary 694 Mycobacterium szulgai infection and treatment in a patient receiving anti-tumor necrosis

696 70. Maloney JM, Gregg CR, Stephens DS, Manian FA, Rimland D. Infections caused by 697 Mycobacterium szulgai in humans. Rev Infect Dis. 1987;9(6):1120-6.

698 71. Anagonou EG, Johnson RC, Barogui YT, Sopoh GE, Ayelo GA, Wadagni AC, et al. 699 Decrease in Mycobacterium ulcerans disease (Buruli ulcer) in the Lalo District of Benin 700 (West Africa). BMC Infect Dis. 2019;19(1):247. 
701 72. Sizaire V, Nackers F, Comte E, Portaels F. Mycobacterium ulcerans infection: control, 702 diagnosis, and treatment. Lancet Infect Dis. 2006;6(5):288-96.

703

704 Acknowledgments

705 We acknowledge Biorepository, BSL-3, and BSL-2 laboratories, qPCR, and DNA-sequencing 706 institutional central core facilities. R.M., S.M., and K.C.M received their fellowships from 707 Ramalingaswami, ILS Flagship, and SERB core research grant, respectively. We would like to 708 thank Dr. Moumita Biswas for scientific input and professional scientific editing of this 709 manuscript. We thank all the volunteers who provided samples for research purposes.

$711 \quad$ Funding

712 The funding for this research was obtained from institute's core funding from the Department of 713 Biotechnology (DBT), Government of India. This work was also supported by the ILS flagship 714 project (BT/ILS/Flagship/2019) from DBT, Ramalingaswami Re-entry fellowship (BT/RLF/Re715 entry/25/2015), and SERB core research grant (CRG/2018/002052).

\section{Author information}

718 Punit Prasad $^{1 * \$}$, Soumendu Mahapatra ${ }^{1 *}$, and Rasmita Mishra ${ }^{*}$, First co-authors

719 Ajay Parida ${ }^{1 \$}$ and Punit Prasad ${ }^{1 * \$}$ are co-corresponding authors 
720 Krushna Chandra Murmu ${ }^{1}$, Shifu Aggarwal ${ }^{1}$, Manisha Sethi ${ }^{1}$, Priyanka Mohapatra ${ }^{1}$, Arup

721 Ghosh $^{1}$, Rina Yadav ${ }^{1}$, Hiren Dodia ${ }^{1}$, Shamima Azma Ansari ${ }^{1}$, Saikat De ${ }^{1}$, Deepak Singh ${ }^{1}$, Amol

722 Suryawanshi ${ }^{1}$, Rupesh Dash ${ }^{1}$, Shantibhushan Senapati ${ }^{1}$, Tushar K. Beuria ${ }^{1}$, Soma

723 Chattopadhyay ${ }^{1}$, Gulam Hussain Syed ${ }^{1}$, Rajeeb Swain ${ }^{1}$, Sunil K. Raghav ${ }^{1}$, Ajay Parida ${ }^{1 \$}$

$724{ }^{1}$ Institute of Life Sciences, Bhubaneswar, Odisha, India.

725

726 Contributions

727 P.P. and A.P. conceptualized the study and secured funding. P.P and S.M. initiated the work,

728 directed overall workflow, interpreted data, and troubleshooted the experiments. R.M. did most

729 of the bioinformatics analysis and S.M., K.C.M. and A.G. helped in bioinformatics analysis and

730 troubleshooting. S.M., S.A., M.S., P.M., R.Y., H.D., S.A.A., S.D., and D.S., helped with the

731 preprocessing of the samples in Biosafety level 3 (BSL3) facility and nucleic acid extractions.

732 S.M., S.A., M.S., P.M., and R.Y. were involved in amplicon library preparations. R.S. provided 733 samples from the Biorepository. A.S., R.D., S.S., T.K.B., S.C., G.H.S., R.S., S.K.R., P.P., and 734 A.P. coordinated with COVID-19 sampling and testing at BSL3. P.P., S.M., and R.M wrote the 735 manuscript.

\section{Ethics declarations}

738 Ethics approval and consent to participate

739 Ethical permission for nasopharyngeal microbiome study and the biorepository was obtained 740 from the Institutional Ethical Committee (IEC)/Institutional Review Board (IRB) of the Institute 
741 of Life Sciences [(102/HEC/2020) and (100/HEC/2020)]. Approval was also obtained from the

742 Institutional Biosafety Committee (IBSC) (V-122-MISC/2007-08/01/2/2.1) for this study and the

743 biorepository (V-122-MISC/2007-08/01) and from the Review Committee on Genetic

744 Manipulations (RCGM) under Department of Biotechnology, Ministry of Science and

745 Technology.

746 Consent for publication

747 All authors have given consent for publication of this manuscript.

748 Competing interest

749 The authors declare no competing commercial or financial interests in relation to this work.

751 Figure Legends:

752 Figure 1: Schema of nasopharyngeal sample processing, 16S sequencing, and OTU-based

753 sample distribution. (A) Flow chart showing nasopharyngeal sample processing for DNA 754 extraction, amplicon library preparation, Oxford Nanopore sequencing, and bioinformatics 755 analysis pipeline. (B) Pie chart showing the nasopharyngeal samples (controls, symptomatic, and 756 asymptomatic) used in this study. (C) t-SNE plot showing the OTU-based sample distribution and 757 ordination points for control, symptomatic, and asymptomatic samples.

Figure 2: Alpha/beta diversities and dysbiosis index in COVID-19-positive and negative nasopharyngeal samples. (A-B) Alpha diversity index (Shannon/Simpson) between control and COVID-19-infected samples (pairwise Wilcoxon rank-sum test $p=\leq 0.05$ ). (C-D) Same analysis 
762 as above where the COVID-19-infected samples are classified as asymptomatic and symptomatic

763 compared to the control group. (E-F) Linear regression model showing the association between

764 total OTU count and Shannon/Simpson diversity index for each sample; the shaded grey region

765 represents $95 \%$ confidence intervals of two groups, symptomatic and asymptomatic, with

766 correlation (Spearman) regression line [Shannon: $\mathrm{R}=-0.35$ (asymptomatic), $\mathrm{R}=-0.54$

767 (symptomatic) and Simpson: $\mathrm{R}=-0.58$ (asymptomatic), $\mathrm{R}=-0.77$ (symptomatic)]. (G) Principal

768 coordinate analysis (PCoA) showing beta diversity in asymptomatic, symptomatic, and control

769 sample groups based on unifrac (weighted/unweighted) distance ( $\mathrm{p}=0.001$, PERMANOVA). (H)

770 Violin plot showing dysbiosis indexes of samples from control, asymptomatic, and symptomatic

771 participants (pairwise Wilcoxon rank-sum test $\mathrm{p}=\leq 0.05$ ).

772

773 Figure 3: Taxonomic classification of bacterial communities using NMDS, correlation, and

774 CCA. (A) NMDS ordination of Bray-Curtis distance matrix based on all samples and bacterial 775 communities of each taxonomy level (phylum, order, family, and genus) (ANOSIM p $=<0.05$ ).

776 (B) The density plot representing the Spearman correlation coefficient at each taxonomy level 777 (phylum, order, family, and genus); dotted line indicates the mean value of each sample group 778 (Kolmogorov-Smirnov (KS) Test $\mathrm{p} \leq$ 0.05). (C) Heatmap of Spearman correlation for genus level 779 with sample correlation (lower) and OTU correlation (upper). Five clusters (C1, C2, C3, C4, and 780 C5) were generated using unsupervised hierarchical clustering from the OTU correlation plot. (D) 781 Sample-wise OTU density plot for each cluster (C1, C2, C3, C4, and C5) showing relative 782 abundance. (E-F) CCA plot of microbial community composition for each cluster and bar plot 783 representing cumulative variation percentage from two components [C1 (92.4\%), C2 (80.5\%), C3 

analysis.

Figure 4: Linear discriminant analysis effect size (LefSe) analysis revealed distinct genus-

level OTUs in control, asymptomatic, and symptomatic participants. (A) Heatmap showing genus level OTU $(\mathrm{n}=181)$ abundance distribution from four clusters $(\mathrm{C} 1, \mathrm{C} 2, \mathrm{C} 4$, and $\mathrm{C} 5)$ identified from CCA analysis in control, asymptomatic and symptomatic samples. The OTUs marked on either side of the heatmap were obtained from one-against-all and all-against-all comparison in LDA analysis (B) The cladogram shows the output of the LEfSe (LDA score >2.0), which identified taxonomic differences between sample groups. Each circle represents a bacterial taxon, and each ring of taxonomy level starting with kingdom in the innermost circle is followed by phylum, class, order, family, and genus in the outermost circle. The different color intensities indicate the different taxonomy levels, and the diameter of each circle is proportional to the taxon's abundance and correlates with the LDA score. (C) The histogram of the LDA scores (score >2.0 and all-against-all) was computed for differentially abundant taxa between sample groups. The effect size of specific taxa in the particular group at the genus level. (D) Histogram of the all LefSespecific taxa (Mycoplasma, Streptosporangium, Citrobacter, Acidocella, Mycolicibacterium, Mycolicibacillus, Mycobacterium, Mycobacteroides, Orientia, Gallibacterium,

802 Cellulosimicrobium, and Oerskovia) showing relative abundance across sample groups. Solid and 803 dotted lines show median and mean relative abundance respectively. (E) Weighted correlation network analysis (WGCNA) was used for network construction and plotted using Gephi. Each 805 node of the network represents the individual bacterial genera with their respective abundance size, and the edges represent correlation strength with edge weight by thickness. The pie chart within 
each node represents abundance for each genus. The dotted line shows two distinct modules

808 (control and infected) created in the network analysis. (F) Violin plot showing the dysbiosis indices 809 of LefSe sample groups (pairwise Wilcoxon rank-sum test p-value < 0.05).

811 Figure 5: Area under the curve-receiver operating characteristic (AUC-ROC) validation and 812 correlation of genera with the symptoms of COVID-19 subjects. (A) ROC curve for LDA 813 classified symptomatic and asymptomatic group. AUC of 0.80 with $95 \%$ confidence interval (CI). 814 (B) Correlation between bacteria at genus level and clinical symptoms of patients. (C-D) ROC 815 curve for chest pain and fever in the symptomatic and asymptomatic groups. The AUCs were 0.904 816 (chest pain) and 0.793 (fever) with 95\% confidence interval (CI).

817 Figure 6: Relative abundance and species-specific OTUs reveal Mycobacterium spp. and 818 Mycoplasma as key opportunistic pathogens. (A) Heatmap showing the taxonomic distribution 819 of 54 species obtained from 12 genus based on LDA scores. (B-D) Boxplots showing relative 820 abundance of opportunistic pathogens (Statistical parameters (Wilcoxon Rank test): $\mathrm{ns}: \mathrm{p}=>0.05$, $821 * \mathrm{p}<=0.05, * * \mathrm{p}<=0.01, * * * \mathrm{p}<=0.001, * * * * \mathrm{p}<=0.0001)$ in $\mathrm{C}$ vs. IA, C vs. IS, and IA vs.

822 IS groups. (E) Spearman correlation showing relationship between opportunistic pathogenic 823 bacteria and patient's clinical symptoms.

824 Table 1: Details of samples included in this study

\begin{tabular}{|l|l|l|l|}
\hline & Control (n=12) & $\begin{array}{l}\text { Asymptomatic } \\
(\mathbf{n = 2 5})\end{array}$ & $\begin{array}{l}\text { Symptomatic } \\
(\mathbf{n = 2 1})\end{array}$ \\
\hline Sex & & & \\
\hline Male & $5(41.66 \%)$ & $18(72 \%)$ & $19(90.47 \%)$ \\
\hline Female & $7(58.33 \%)$ & $7(28 \%)$ & $2(9.52 \%)$ \\
\hline
\end{tabular}




\begin{tabular}{|l|l|l|l|}
\hline Age (years) & 31 (median) & 26 (median) & 32 (median) \\
\hline Symptoms & & & \\
\hline Dry Cough & NA & NA & $7(33.3 \%)$ \\
\hline Fever & NA & NA & $17(80.95 \%)$ \\
\hline Tiredness & NA & NA & $8(38.09 \%)$ \\
\hline Sore throat & NA & NA & $11(52.38 \%)$ \\
\hline Body pain & NA & NA & $13(61.9 \%)$ \\
\hline Chest pain & NA & NA & $9(42.85 \%)$ \\
\hline Fever + Body pain & NA & NA & $4(19.04 \%)$ \\
\hline Fever + multiple symptoms* & NA & NA & $16(76.19 \%)$ \\
\hline Fever + chest pain & NA & NA & $8(38.09 \%)$ \\
\hline $\begin{array}{l}\text { Loss of smell/taste + multiple } \\
\text { symptoms* without fever }\end{array}$ & NA & NA & $2(9.52 \%)$ \\
\hline
\end{tabular}

*Multiple symptoms refer to having more than one symptoms from symptoms list.

826 Table 2: Phylum based on relative abundance and their respective values

\begin{tabular}{|l|l|l|l|l|l|l|}
\hline Phylum & Mean & $\begin{array}{l}\text { 1st } \\
\text { Quartile }\end{array}$ & Median & $\begin{array}{l}\text { 3rd } \\
\text { Quartile }\end{array}$ & p_value & BH_FDR \\
\hline Proteobacteria & $1.51 \mathrm{E}-01$ & $3.79 \mathrm{E}-02$ & $4.53 \mathrm{E}-02$ & $7.29 \mathrm{E}-02$ & $6.56 \mathrm{E}-07$ & $6.56 \mathrm{E}-07$ \\
\hline Fusobacteria & $2.25 \mathrm{E}-03$ & $1.31 \mathrm{E}-03$ & $1.72 \mathrm{E}-03$ & $2.71 \mathrm{E}-03$ & $9.00 \mathrm{E}-07$ & $9.00 \mathrm{E}-07$ \\
\hline Actinobacteria & $6.83 \mathrm{E}-01$ & $7.09 \mathrm{E}-01$ & $7.62 \mathrm{E}-01$ & $7.99 \mathrm{E}-01$ & $9.96 \mathrm{E}-07$ & $9.96 \mathrm{E}-07$ \\
\hline Bacteroidetes & $8.77 \mathrm{E}-03$ & $5.71 \mathrm{E}-03$ & $6.66 \mathrm{E}-03$ & $1.00 \mathrm{E}-02$ & $1.48 \mathrm{E}-06$ & $1.48 \mathrm{E}-06$ \\
\hline Tenericutes & $9.37 \mathrm{E}-04$ & $1.35 \mathrm{E}-04$ & $5.60 \mathrm{E}-04$ & $1.21 \mathrm{E}-03$ & $2.21 \mathrm{E}-06$ & $2.21 \mathrm{E}-06$ \\
\hline Chloroflexi & $2.25 \mathrm{E}-03$ & $6.47 \mathrm{E}-04$ & $9.84 \mathrm{E}-04$ & $1.46 \mathrm{E}-03$ & $6.54 \mathrm{E}-06$ & $6.54 \mathrm{E}-06$ \\
\hline Chlamydiae & $5.93 \mathrm{E}-04$ & $2.82 \mathrm{E}-04$ & $4.98 \mathrm{E}-04$ & $7.76 \mathrm{E}-04$ & $7.27 \mathrm{E}-06$ & $7.27 \mathrm{E}-06$ \\
\hline Fibrobacteres & $5.65 \mathrm{E}-04$ & $2.86 \mathrm{E}-04$ & $4.05 \mathrm{E}-04$ & $7.10 \mathrm{E}-04$ & $4.77 \mathrm{E}-05$ & $4.77 \mathrm{E}-05$ \\
\hline Thermodesulfobacteria & $2.62 \mathrm{E}-02$ & $2.09 \mathrm{E}-02$ & $2.84 \mathrm{E}-02$ & $3.24 \mathrm{E}-02$ & $8.13 \mathrm{E}-04$ & $8.13 \mathrm{E}-04$ \\
\hline Aquificae & $2.31 \mathrm{E}-04$ & $1.19 \mathrm{E}-04$ & $2.30 \mathrm{E}-04$ & $2.93 \mathrm{E}-04$ & $1.59 \mathrm{E}-03$ & $1.59 \mathrm{E}-03$ \\
\hline Firmicutes & $1.23 \mathrm{E}-01$ & $1.19 \mathrm{E}-01$ & $1.28 \mathrm{E}-01$ & $1.44 \mathrm{E}-01$ & $4.65 \mathrm{E}-02$ & $4.65 \mathrm{E}-02$ \\
\hline
\end{tabular}


Chlorobi

1.02E-03

6.81E-04

9.32E-04

1.27E-03

$8.58 \mathrm{E}-01$

$8.58 \mathrm{E}-01$

828 Table 3: Top 30 family based on relative abundance and their respective values

\begin{tabular}{|c|c|c|c|c|c|c|}
\hline Family & Mean & 1st quartile & Median & \begin{tabular}{|l|} 
3rd \\
quartile
\end{tabular} & p_value & BH_FDR \\
\hline Acetobacteraceae & $5.08 \mathrm{E}-02$ & $5.00 \mathrm{E}-02$ & $5.12 \mathrm{E}-02$ & $5.28 \mathrm{E}-02$ & 9.14E-07 & 9.14E-07 \\
\hline Actinomycetaceae & $2.23 \mathrm{E}-02$ & $2.10 \mathrm{E}-02$ & $2.18 \mathrm{E}-02$ & $2.32 \mathrm{E}-02$ & $8.18 \mathrm{E}-03$ & $8.18 \mathrm{E}-03$ \\
\hline Aeromonadaceae & $2.96 \mathrm{E}-02$ & $2.54 \mathrm{E}-02$ & $3.07 \mathrm{E}-02$ & $3.38 \mathrm{E}-02$ & $8.18 \mathrm{E}-03$ & $8.18 \mathrm{E}-03$ \\
\hline Alcaligenaceae & $8.14 \mathrm{E}-02$ & $8.50 \mathrm{E}-02$ & $8.71 \mathrm{E}-02$ & $9.43 \mathrm{E}-02$ & $1.41 \mathrm{E}-06$ & $1.41 \mathrm{E}-06$ \\
\hline Bifidobacteriaceae & $7.69 \mathrm{E}-02$ & $4.59 \mathrm{E}-02$ & $6.52 \mathrm{E}-02$ & $1.04 \mathrm{E}-01$ & $2.99 \mathrm{E}-03$ & $2.99 \mathrm{E}-03$ \\
\hline Brevibacteriaceae & $4.37 \mathrm{E}-02$ & $2.72 \mathrm{E}-02$ & $3.82 \mathrm{E}-02$ & $5.98 \mathrm{E}-02$ & $6.35 \mathrm{E}-04$ & $6.35 \mathrm{E}-04$ \\
\hline Cellulomonadaceae & $6.75 \mathrm{E}-02$ & $4.60 \mathrm{E}-02$ & $6.29 \mathrm{E}-02$ & $8.52 \mathrm{E}-02$ & $8.64 \mathrm{E}-05$ & $8.64 \mathrm{E}-05$ \\
\hline Chromobacteriaceae & $2.30 \mathrm{E}-02$ & $2.27 \mathrm{E}-02$ & $2.30 \mathrm{E}-02$ & $2.32 \mathrm{E}-02$ & $9.37 \mathrm{E}-07$ & $9.37 \mathrm{E}-07$ \\
\hline Corynebacteriaceae & $5.49 \mathrm{E}-02$ & $4.18 \mathrm{E}-02$ & $5.05 \mathrm{E}-02$ & $6.52 \mathrm{E}-02$ & $2.36 \mathrm{E}-05$ & $2.36 \mathrm{E}-05$ \\
\hline Enterobacteriaceae & $1.26 \mathrm{E}-01$ & $3.61 \mathrm{E}-02$ & $1.65 \mathrm{E}-01$ & $2.17 \mathrm{E}-01$ & $1.73 \mathrm{E}-06$ & $1.73 \mathrm{E}-06$ \\
\hline Erwiniaceae & $3.19 \mathrm{E}-02$ & $2.97 \mathrm{E}-02$ & $3.10 \mathrm{E}-02$ & $3.46 \mathrm{E}-02$ & $2.01 \mathrm{E}-06$ & $2.01 \mathrm{E}-06$ \\
\hline Erysipelotrichaceae & $2.26 \mathrm{E}-02$ & $2.21 \mathrm{E}-02$ & $2.26 \mathrm{E}-02$ & $2.30 \mathrm{E}-02$ & $1.69 \mathrm{E}-06$ & $1.69 \mathrm{E}-06$ \\
\hline Eubacteriaceae & $2.99 \mathrm{E}-02$ & $2.58 \mathrm{E}-02$ & $2.84 \mathrm{E}-02$ & $3.28 \mathrm{E}-02$ & $1.85 \mathrm{E}-06$ & $1.85 \mathrm{E}-06$ \\
\hline Lactobacillaceae & $2.12 \mathrm{E}-02$ & $2.05 \mathrm{E}-02$ & $2.10 \mathrm{E}-02$ & $2.18 \mathrm{E}-02$ & 5.39E-03 & $5.39 \mathrm{E}-03$ \\
\hline Micrococcaceae & $3.52 \mathrm{E}-02$ & $2.65 \mathrm{E}-02$ & $3.37 \mathrm{E}-02$ & $4.36 \mathrm{E}-02$ & $3.49 \mathrm{E}-03$ & $3.49 \mathrm{E}-03$ \\
\hline Micromonosporaceae & $2.41 \mathrm{E}-02$ & $2.25 \mathrm{E}-02$ & $2.43 \mathrm{E}-02$ & $2.53 \mathrm{E}-02$ & $2.65 \mathrm{E}-06$ & $2.65 \mathrm{E}-06$ \\
\hline Morganellaceae & $5.01 \mathrm{E}-02$ & $4.51 \mathrm{E}-02$ & $5.30 \mathrm{E}-02$ & $5.93 \mathrm{E}-02$ & $1.90 \mathrm{E}-05$ & $1.90 \mathrm{E}-05$ \\
\hline Mycobacteriaceae & $2.19 \mathrm{E}-01$ & $1.85 \mathrm{E}-01$ & $2.42 \mathrm{E}-01$ & $2.69 \mathrm{E}-01$ & $7.93 \mathrm{E}-07$ & $7.93 \mathrm{E}-07$ \\
\hline Neisseriaceae & $5.24 \mathrm{E}-02$ & $5.08 \mathrm{E}-02$ & $5.23 \mathrm{E}-02$ & $5.43 \mathrm{E}-02$ & $1.29 \mathrm{E}-06$ & $1.29 \mathrm{E}-06$ \\
\hline Nocardiaceae & $3.15 \mathrm{E}-02$ & $2.46 \mathrm{E}-02$ & $3.41 \mathrm{E}-02$ & $3.63 \mathrm{E}-02$ & $8.90 \mathrm{E}-07$ & $8.90 \mathrm{E}-07$ \\
\hline Pasteurellaceae & $4.73 \mathrm{E}-02$ & $3.34 \mathrm{E}-02$ & $5.14 \mathrm{E}-02$ & $5.75 \mathrm{E}-02$ & $5.89 \mathrm{E}-05$ & $5.89 \mathrm{E}-05$ \\
\hline Pectobacteriaceae & $2.17 \mathrm{E}-02$ & $2.05 \mathrm{E}-02$ & $2.15 \mathrm{E}-02$ & $2.27 \mathrm{E}-02$ & $2.09 \mathrm{E}-06$ & $2.09 \mathrm{E}-06$ \\
\hline
\end{tabular}




\begin{tabular}{|l|l|l|l|l|l|l|}
\hline Peptostreptococcaceae & $3.01 \mathrm{E}-02$ & $2.56 \mathrm{E}-02$ & $2.82 \mathrm{E}-02$ & $3.23 \mathrm{E}-02$ & $1.89 \mathrm{E}-06$ & $1.89 \mathrm{E}-06$ \\
\hline Propionibacteriaceae & $1.17 \mathrm{E}-01$ & $9.00 \mathrm{E}-02$ & $1.20 \mathrm{E}-01$ & $1.33 \mathrm{E}-01$ & $8.90 \mathrm{E}-07$ & $8.90 \mathrm{E}-07$ \\
\hline Pseudonocardiaceae & $2.94 \mathrm{E}-02$ & $2.70 \mathrm{E}-02$ & $2.98 \mathrm{E}-02$ & $3.27 \mathrm{E}-02$ & $1.44 \mathrm{E}-06$ & $1.44 \mathrm{E}-06$ \\
\hline
\end{tabular}

830 Table 4: Mean and median value of density plots.

\begin{tabular}{|l|l|l|l|l|}
\hline Taxonomic rank & Statistic value & Control & Asymptomatic & Symptomatic \\
\hline \multirow{4}{*}{ Phylum } & Mean & $8.96 \mathrm{E}-01$ & $8.68 \mathrm{E}-01$ & $8.90 \mathrm{E}-01$ \\
\cline { 2 - 5 } & Median & $9.30 \mathrm{E}-01$ & $8.95 \mathrm{E}-01$ & $9.16 \mathrm{E}-01$ \\
\hline \multirow{3}{*}{ Order } & Mean & $8.58 \mathrm{E}-01$ & $7.70 \mathrm{E}-01$ & $8.36 \mathrm{E}-01$ \\
\cline { 2 - 5 } Family & Median & $8.78 \mathrm{E}-01$ & $8.21 \mathrm{E}-01$ & $8.93 \mathrm{E}-01$ \\
\hline \multirow{3}{*}{ Genus } & Mean & $8.22 \mathrm{E}-01$ & $6.86 \mathrm{E}-01$ & $7.60 \mathrm{E}-01$ \\
\cline { 2 - 5 } & Median & $8.63 \mathrm{E}-01$ & $7.46 \mathrm{E}-01$ & $8.06 \mathrm{E}-01$ \\
\cline { 2 - 5 } & Mean & $7.95 \mathrm{E}-01$ & $5.65 \mathrm{E}-01$ & $6.51 \mathrm{E}-01$ \\
\cline { 2 - 5 } & Median & $6.39 \mathrm{E}-01$ & $8.33 \mathrm{E}-01$ & $7.01 \mathrm{E}-01$ \\
\hline
\end{tabular}

Table 5: Result of Kolmogorov-Smirnov (KS) test between the densities of each taxonomic 832 rank.

\begin{tabular}{|l|l|l|l|}
\hline Taxonomic rank & $\begin{array}{l}\text { Control vs } \\
\text { Asymptomatic }\end{array}$ & $\begin{array}{l}\text { Control vs } \\
\text { Symptomatic }\end{array}$ & $\begin{array}{l}\text { Asymptomatic vs } \\
\text { Symptomatic }\end{array}$ \\
\hline \multirow{4}{*}{ Phylum } & $\mathrm{D}=1.88 \mathrm{e}-01$ & $\mathrm{D}=1.07 \mathrm{e}-01$ & $\mathrm{D}=1.01 \mathrm{e}-01$ \\
\cline { 2 - 4 } & $\mathrm{p}$-value $=3.03 \mathrm{e}-02$ & $\mathrm{p}$-value $=0.4834$ & $\mathrm{p}$-value $=9.78 \mathrm{e}-04$ \\
\hline \multirow{3}{*}{ Frder } & $\mathrm{D}=3.18 \mathrm{e}-01$ & $\mathrm{D}=9.97 \mathrm{e}-02$ & $\mathrm{D}=2.30 \mathrm{e}-01$ \\
\cline { 2 - 4 } & $\mathrm{p}$-value $=1.237 \mathrm{e}-05$ & $\mathrm{p}$-value $=0.5706$ & $\mathrm{p}$-value $<2.2 \mathrm{e}-16$ \\
\hline \multirow{3}{*}{ Genus } & $\mathrm{D}=4.14 \mathrm{e}-01$ & $\mathrm{D}=2.93 \mathrm{e}-01$ & $\mathrm{D}=2.25 \mathrm{e}-01$ \\
\cline { 2 - 4 } & $\mathrm{p}$-value $=2.706 \mathrm{e}-09$ & $\mathrm{p}$-value $=4.75 \mathrm{e}-05$ & $\mathrm{p}$-value $<2.2 \mathrm{e}-16$ \\
\hline & $\mathrm{D}=5.94 \mathrm{e}-01$ & $\mathrm{D}=5.06 \mathrm{e}-01$ & $\mathrm{D}=2.28 \mathrm{e}-01$ \\
\cline { 2 - 4 } & $\mathrm{p}$-value $<2.2 \mathrm{e}-16$ & $\mathrm{p}$-value $=3.308 \mathrm{e}-14$ & $\mathrm{p}$-value $<2.2 \mathrm{e}-16$ \\
\hline
\end{tabular}

Table 6: Linear discriminate analysis (LDA) score for all-against-all analysis 


\begin{tabular}{|l|l|l|l|l|}
\hline Genus & $\begin{array}{l}\text { Highest mean } \\
\text { among all the } \\
\text { classes }\end{array}$ & Samples & $\begin{array}{l}\text { LDA score (log } \\
\mathbf{1 0})\end{array}$ & pvalue \\
\hline Oerskovia & 3.83 & Asymptomatic & 3.44 & $1.96 \mathrm{E}-02$ \\
\hline Cellulosimicrobium & 3.85 & Asymptomatic & 3.5 & $1.73 \mathrm{E}-02$ \\
\hline Gallibacterium & 3.84 & Control & 3.36 & $8.46 \mathrm{E}-04$ \\
\hline Orientia & 3.88 & Control & 3.46 & $3.07 \mathrm{E}-04$ \\
\hline Acidocella & 4.07 & Control & 3.81 & $4.68 \mathrm{E}-07$ \\
\hline Citrobacter & 4.08 & Control & 3.89 & $2.82 \mathrm{E}-07$ \\
\hline Mycobacteroides & 3.88 & Symptomatic & 3.61 & $3.19 \mathrm{E}-07$ \\
\hline Mycolicibacillus & 3.98 & Symptomatic & 3.67 & $3.19 \mathrm{E}-07$ \\
\hline Mycolicibacterium & 3.93 & Symptomatic & 3.71 & $2.55 \mathrm{E}-07$ \\
\hline Mycobacterium & 3.9 & Symptomatic & 3.79 & $2.55 \mathrm{E}-07$ \\
\hline Streptosporangium & 4.16 & Symptomatic & 3.83 & $1.65 \mathrm{E}-04$ \\
\hline Mycoplasma & 4.26 & Symptomatic & 3.95 & $1.64 \mathrm{E}-06$ \\
\hline
\end{tabular}

835 Table 7: WGCNA network data table

\begin{tabular}{|c|c|c|c|c|c|c|c|c|c|c|}
\hline 且 & 堷 & $?_{0}^{n}$ & $\stackrel{\mathscr{O}}{\Xi}$ & 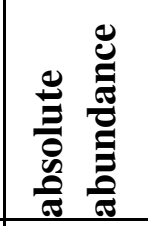 & 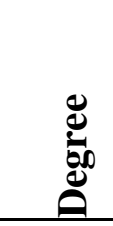 & 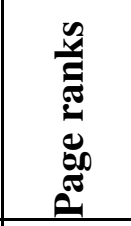 & 总 & 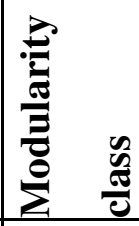 & 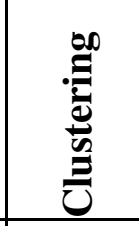 & 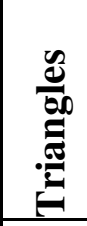 \\
\hline 1 & Acidocella & 525 & M1 & 1362 & 5 & 0.0673 & 0.4963 & 0 & 1 & 10 \\
\hline 2 & Citrobacter & 546 & M1 & 3463 & 5 & 0.0673 & 0.4963 & 0 & 1 & 10 \\
\hline 3 & Gallibacterium & 750 & M1 & 285 & 5 & 0.0673 & 0.4963 & 0 & 1 & 10 \\
\hline 4 & Orientia & 784 & M1 & 263 & 5 & 0.0673 & 0.4963 & 0 & 1 & 10 \\
\hline 5 & $\begin{array}{l}\text { Cellulosimicrobiu } \\
m\end{array}$ & 1710 & M2 & 11832 & 10 & 0.1202 & 1 & 1 & 0.5555 & 25 \\
\hline 6 & Oerskovia & 1713 & M2 & 13210 & 10 & 0.1202 & 1 & 1 & 0.5555 & 25 \\
\hline 7 & Mycobacterium & 1763 & M2 & 139017 & 7 & 0.0852 & 0.8208 & 1 & 0.9047 & 19 \\
\hline 8 & $\begin{array}{l}\text { Mycolicibacteriu } \\
m\end{array}$ & 1766 & M2 & 81430 & 7 & 0.0852 & 0.8208 & 1 & 0.9047 & 19 \\
\hline
\end{tabular}




\begin{tabular}{|l|l|l|l|l|l|l|l|l|l|l|}
\hline 9 & Mycobacteroides & 1774 & $\mathrm{M} 2$ & 7172 & 7 & 0.0852 & 0.8208 & 1 & 0.9047 & 19 \\
\hline 10 & Mycolicibacillus & 1798 & $\mathrm{M} 2$ & 2749 & 7 & 0.0852 & 0.8208 & 1 & 0.9047 & 19 \\
\hline 11 & $\begin{array}{l}\text { Streptosporangiu } \\
m\end{array}$ & 2002 & $\mathrm{M} 2$ & 163 & 5 & 0.0642 & 0.5732 & 1 & 1 & 10 \\
\hline 12 & Mycoplasma & 2100 & $\mathrm{M} 2$ & 475 & 7 & 0.0852 & 0.8208 & 1 & 0.9047 & 19 \\
\hline
\end{tabular}

836

837 Table 8: List of opportunistic pathogenic bacteria

\begin{tabular}{|c|l|l|c|}
\hline $\begin{array}{l}\text { Sl. } \\
\text { No. }\end{array}$ & Species & Clinical manifestation & References \\
\hline 1 & Mycoplasma pneumoniae & $\begin{array}{l}\text { Mycoplasma pneumonia, a form of } \\
\text { atypical bacterial pneumonia. }\end{array}$ & $(42,44,49)$ \\
\hline 2 & Mycobacterium tuberculosis & Tuberculosis (TB) & $(35,38-41)$ \\
\hline 3 & Mycobacterium avium & MAC lung disease & $(52,53)$ \\
\hline 4 & Mycobacterium intracellulare & MAC lung disease & $(54,55)$ \\
\hline 5 & Mycobacterium leprae & Hansen's disease (Leprosy) & $(56,57)$ \\
\hline 6 & Mycobacterium Kansasii & Chronic pulmonary infection & $(58,59)$ \\
\hline 7 & Mycobacterium simiae & Pulmonary infection & $(60,61)$ \\
\hline 8 & Mycobacterium xenopi & NTM lung disease & $(62-64)$ \\
\hline 9 & Mycobacterium gordonae & Pulmonary infection & $(65,66)$ \\
\hline 10 & Mycobacterium asiaticum & Pulmonary and extrapulmonary disease & $(67,68)$ \\
\hline 11 & Mycobacterium szulgai & Pulmonary infection & $(69,70)$ \\
\hline 12 & Mycobacterium ulcerans & Buruli ulcer & $(71,72)$ \\
\hline
\end{tabular}




\section{Figures}

Figure 1

A

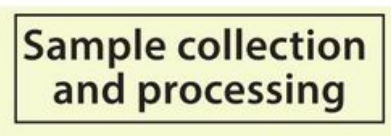

Biorepository Control

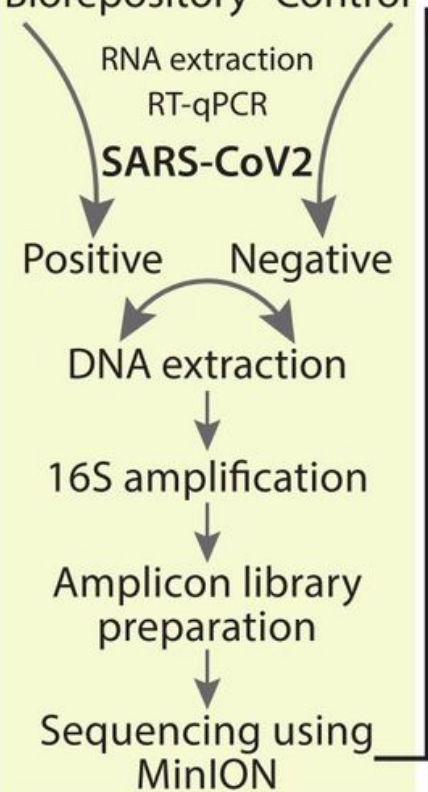

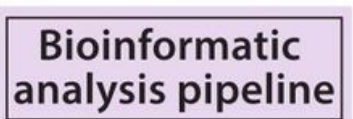

Sequencing data

Fast5 format

Base calling by

Guppy

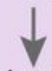

Fastq files

Trimming by

Nanofilt

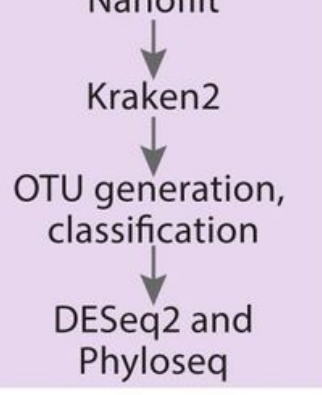

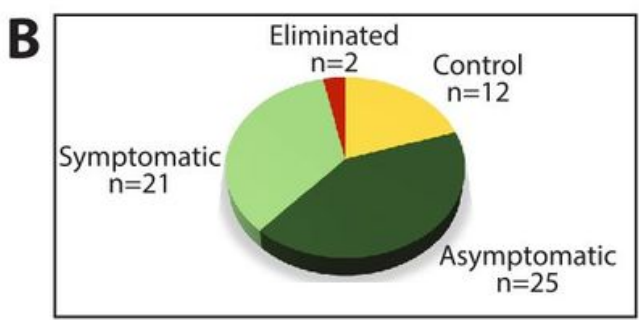

C

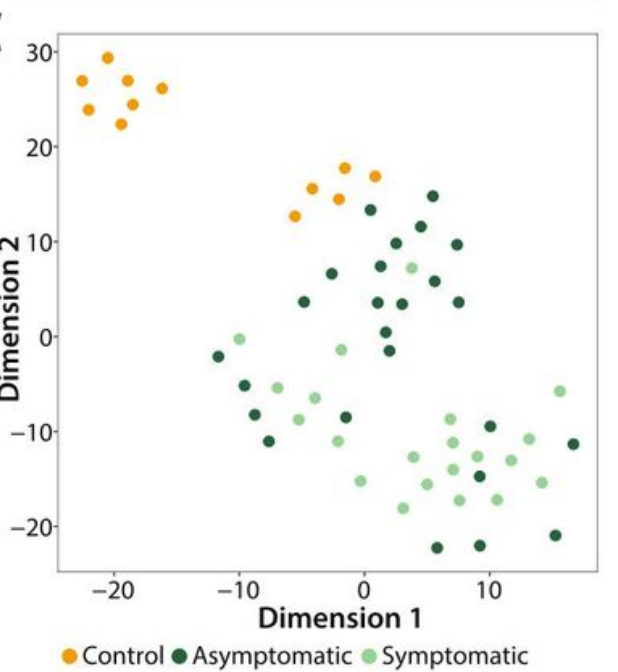

\section{Figure 1}

Schema of nasopharyngeal sample processing, 16S sequencing, and OTU-based sample distribution. (A) Flow chart showing nasopharyngeal sample processing for DNA extraction, amplicon library preparation, Oxford Nanopore sequencing, and bioinformatics analysis pipeline. (B) Pie chart showing the nasopharyngeal samples (controls, symptomatic, and asymptomatic) used in this study. (C) t-SNE plot showing the OTU-based sample distribution and ordination points for control, symptomatic, and asymptomatic samples. 


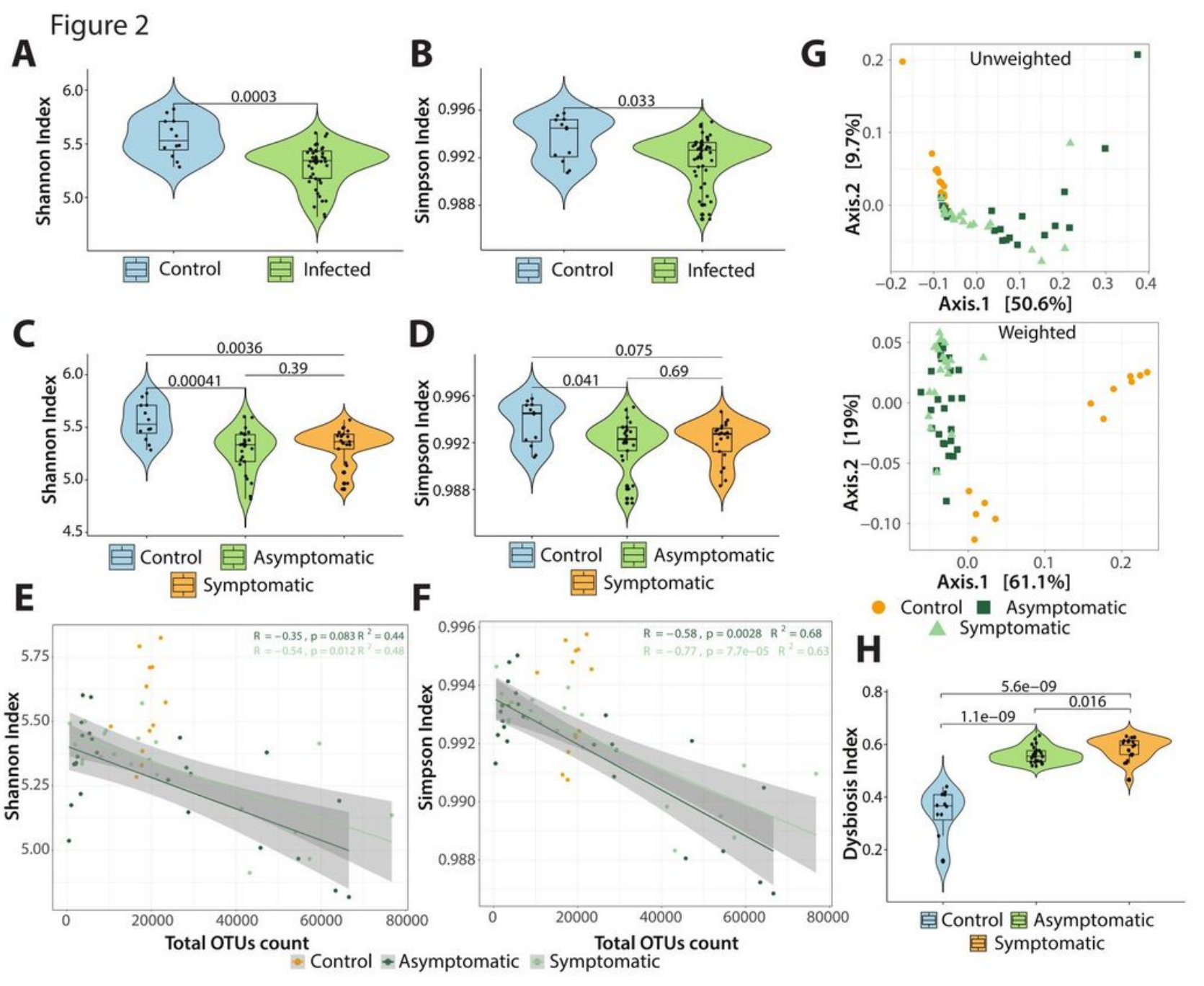

\section{Figure 2}

Alpha/beta diversities and dysbiosis index in COVID-19-positive and negative 760 nasopharyngeal samples. (A-B) Alpha diversity index (Shannon/Simpson) between control and COVID-19-infected samples (pairwise Wilcoxon rank-sum test $p=\leq 0.05$ ). (C-D) Same analysis 35 as above where the COVID19-infected samples are classified as asymptomatic and symptomatic compared to the control group. (EF) Linear regression model showing the association between total OTU count and Shannon/Simpson diversity index for each sample; the shaded grey region represents $95 \%$ confidence intervals of two groups, symptomatic and asymptomatic, with correlation (Spearman) regression line [Shannon: $\mathrm{R}=-0.35$ (asymptomatic), $\mathrm{R}=-0.54767$ (symptomatic) and Simpson: $\mathrm{R}=-0.58$ (asymptomatic), $\mathrm{R}=-0.77$ (symptomatic)]. (G) Principal coordinate analysis (PCoA) showing beta diversity in asymptomatic, symptomatic, and control sample groups based on unifrac (weighted/unweighted) distance $(p=0.001$, PERMANOVA). (H) Violin plot showing dysbiosis indexes of samples from control, asymptomatic, and symptomatic participants (pairwise Wilcoxon rank-sum test $p=\leq 0.05$ ). 

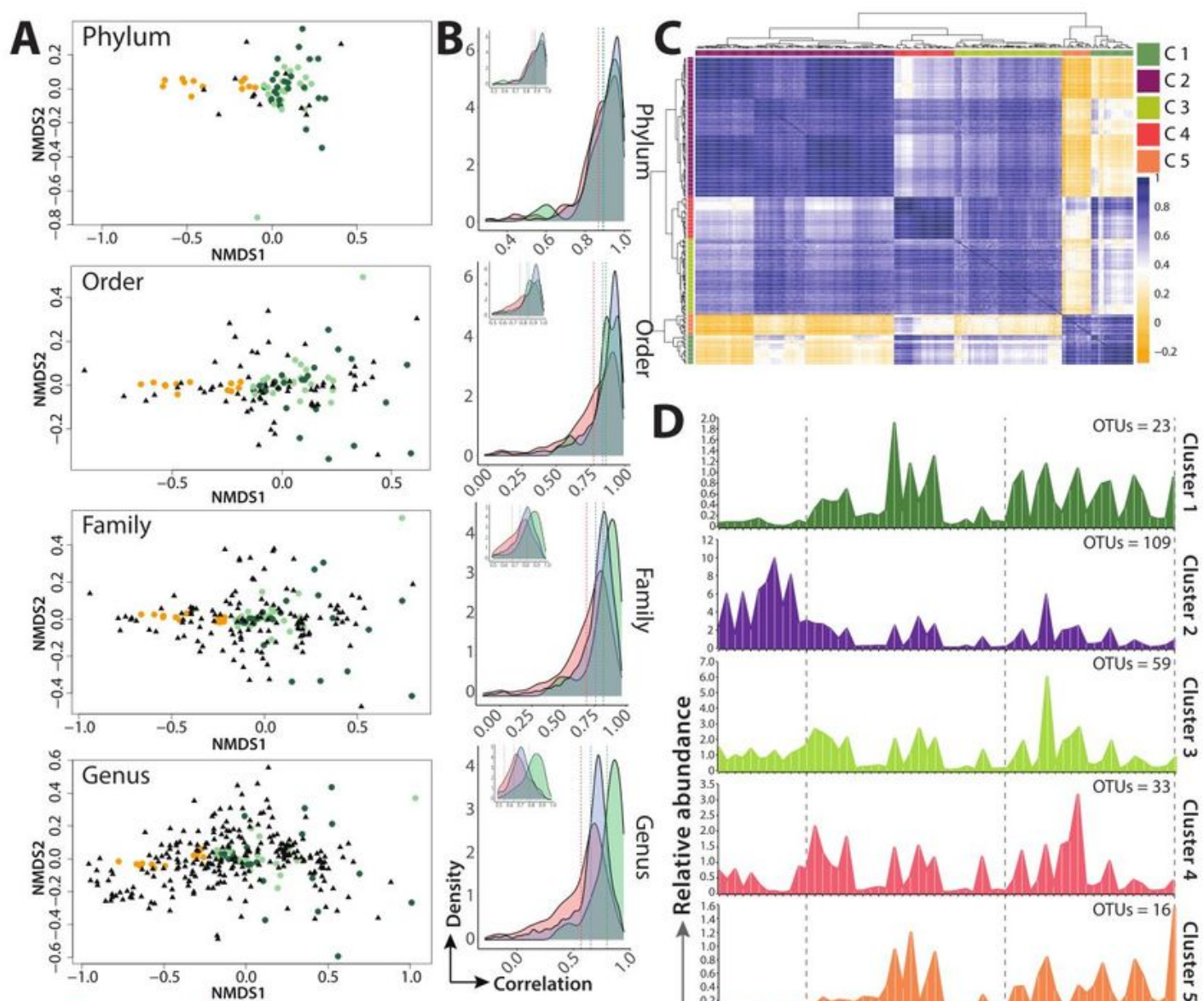

- Control - Asymptomatic

Symptomatic $\boldsymbol{\Delta}$ OTUs

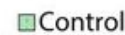

$\square$ Asymptomatic 口Symptomatic
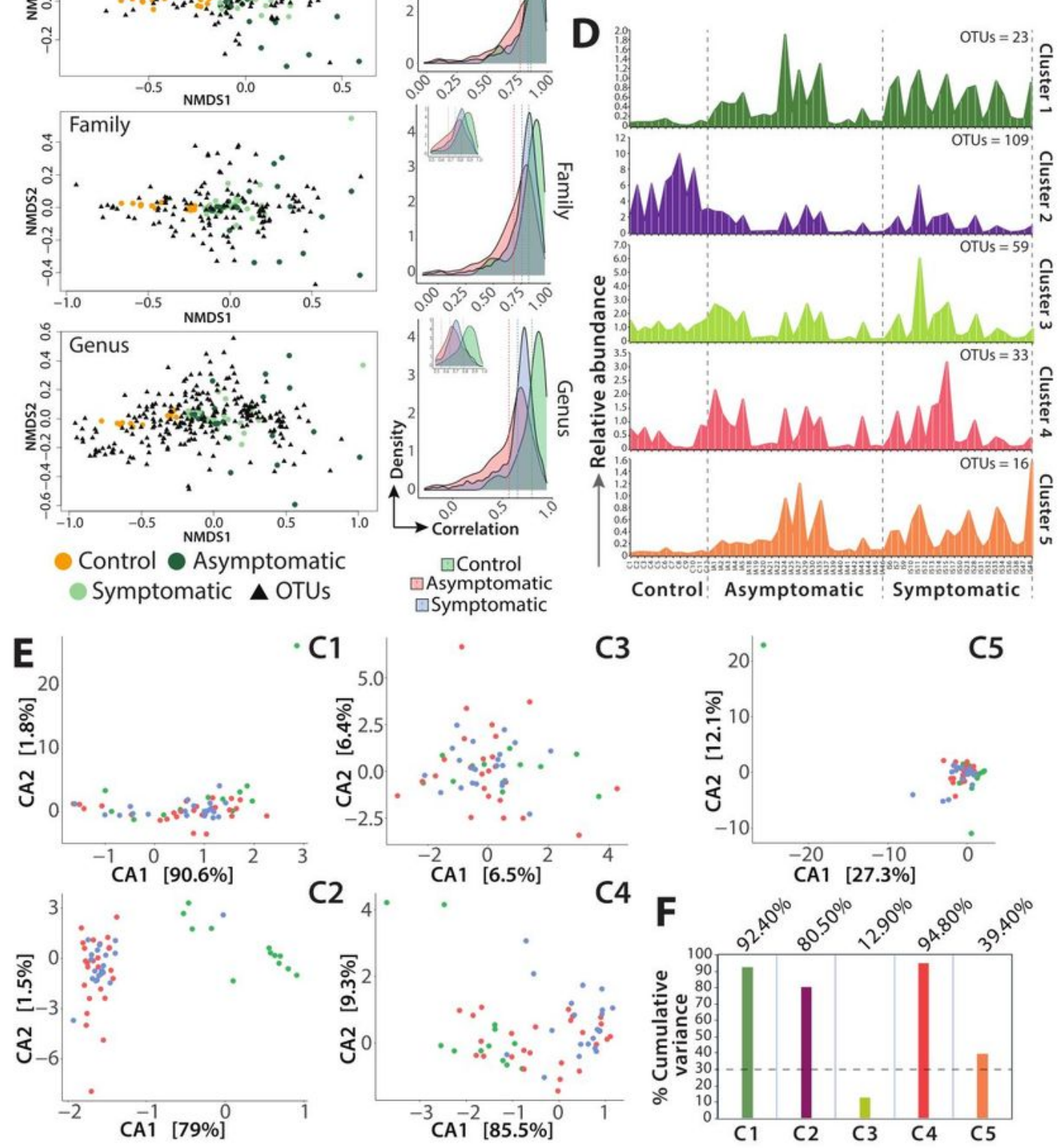

\section{Figure 3}

Taxonomic classification of bacterial communities using NMDS, correlation, andCCA. (A) NMDS ordination of Bray-Curtis distance matrix based on all samples and bacterial communities of each taxonomy level (phylum, order, family, and genus) (ANOSIM $p=<0.05$ ). (B) The density plot representing the Spearman correlation coefficient at each taxonomy level (phylum, order, family, and genus); dotted line indicates the mean value of each sample group (Kolmogorov-Smirnov (KS) Test $p \leq 0.05)$. (C) 
Heatmap of Spearman correlation for genus level with sample correlation (lower) and OTU correlation (upper). Five clusters (C1, C2, C3, C4, and C5) were generated using unsupervised hierarchical clustering from the OTU correlation plot. (D) Sample-wise OTU density plot for each cluster (C1, C2, C3, C4, and C5) showing relative abundance. (E-F) CCA plot of microbial community composition for each cluster and bar plot representing cumulative variation percentage from two components [C1 (92.4\%), C2 (80.5\%), C3 36 $784(12.9 \%)$, C4 (94.8\%), and C5 (39.4\%)]. Dotted line shows 30\% variance cut-off for downstream analysis.
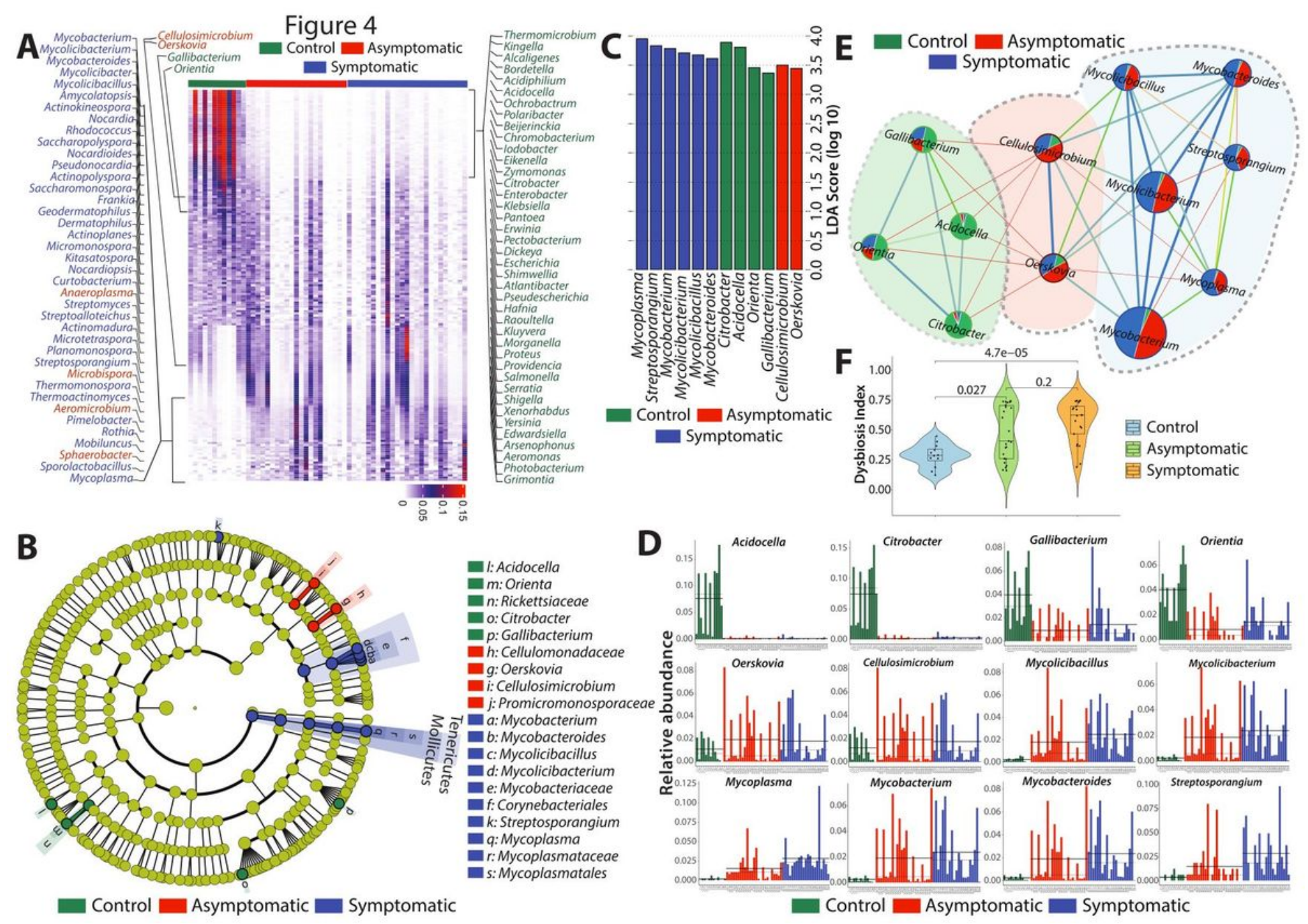

\section{Figure 4}

Linear discriminant analysis effect size (LefSe) analysis revealed distinct genus level OTUs in control, asymptomatic, and symptomatic participants. (A) Heatmap showing genus level OTU ( $n=181)$ abundance distribution from four clusters (C1, C2, C4, and C5) identified from CCA analysis in control, asymptomatic and symptomatic samples. The OTUs marked on either side of the heatmap were obtained from one-against-all and all-against-all comparison in LDA analysis (B) The cladogram shows the output of the LEfSe (LDA score >2.0), which identified taxonomic differences between sample groups. Each circle represents a bacterial taxon, and each ring of taxonomy level starting with kingdom in the innermost circle is followed by phylum, class, order, family, and genus in the outermost circle. The 
different color intensities indicate the different taxonomy levels, and the diameter of each circle is proportional to the taxon's abundance and correlates with the LDA score. (C) The histogram of the LDA scores (score $>2.0$ and all-against-all) was computed for differentially abundant taxa between sample groups. The effect size of specific taxa in the particular group at the genus level. (D) Histogram of the all LefSe specific taxa (Mycoplasma, Streptosporangium, Citrobacter, Acidocella, Mycolicibacterium, Mycolicibacillus, Mycobacterium, Mycobacteroides, Orientia, Gallibacterium, Cellulosimicrobium, and Oerskovia) showing relative abundance across sample groups. Solid and dotted lines show median and mean relative abundance respectively. (E) Weighted correlation network analysis (WGCNA) was used for network construction and plotted using Gephi. Each node of the network represents the individual bacterial genera with their respective abundance size, and the edges represent correlation strength with edge weight by thickness. The pie chart within 37 each node represents abundance for each genus. The dotted line shows two distinct modules (control and infected) created in the network analysis. (F) Violin plot showing the dysbiosis indices of LefSe sample groups (pairwise Wilcoxon rank-sum test p-value < 0.05).

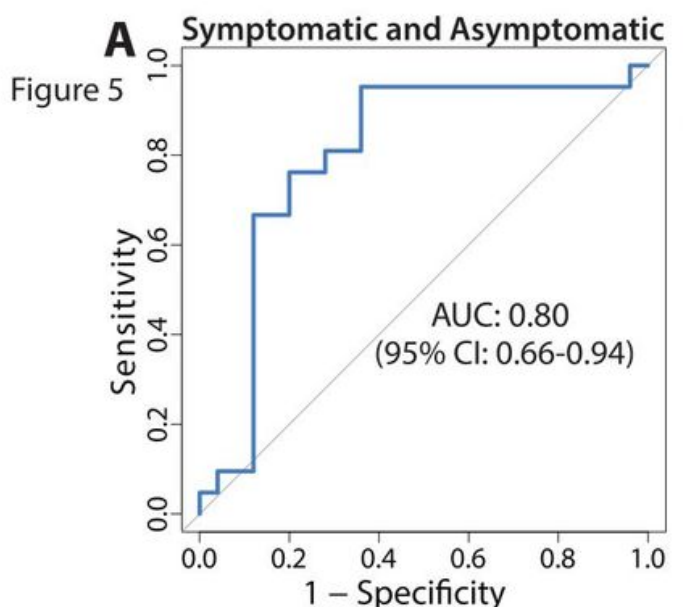

C

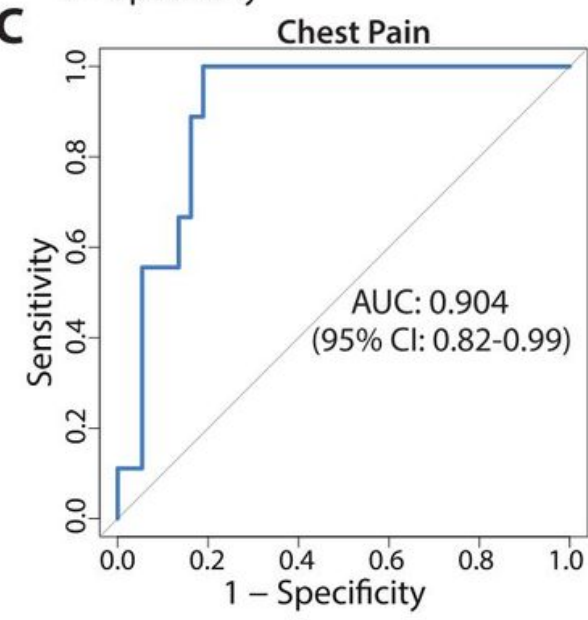

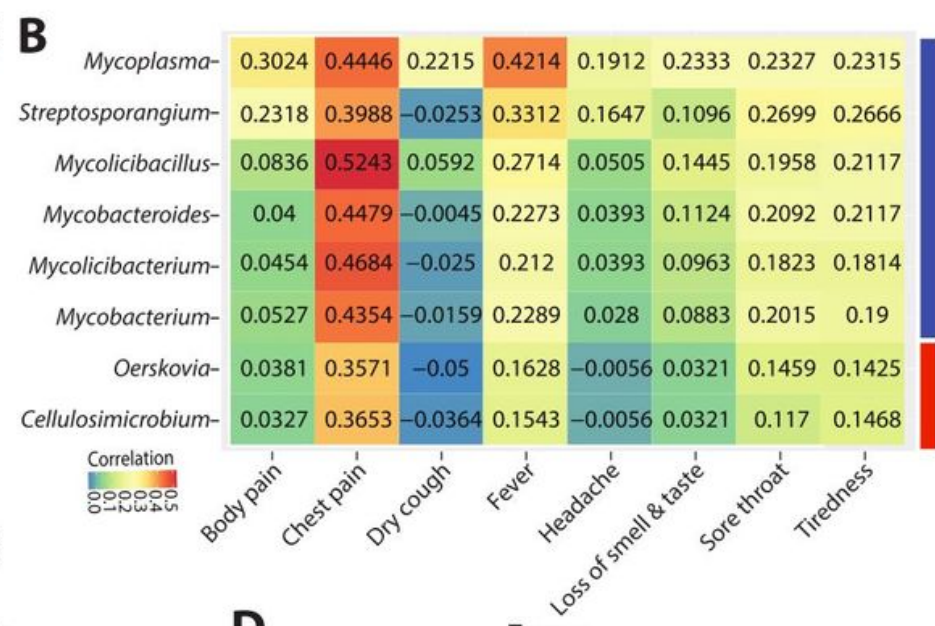

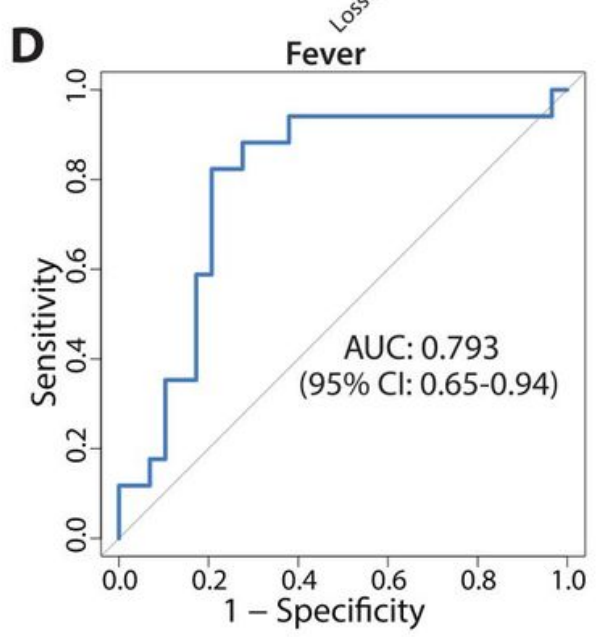

\section{Figure 5}

Area under the curve-receiver operating characteristic (AUC-ROC) validation and correlation of genera with the symptoms of COVID-19 subjects. (A) ROC curve for LDA classified symptomatic and asymptomatic group. AUC of 0.80 with $95 \%$ confidence interval (CI). (B) Correlation between bacteria at genus level and 
clinical symptoms of patients. (C-D) ROC curve for chest pain and fever in the symptomatic and asymptomatic groups. The AUCs were 0.904 (chest pain) and 0.793 (fever) with 95\% confidence interval (Cl).
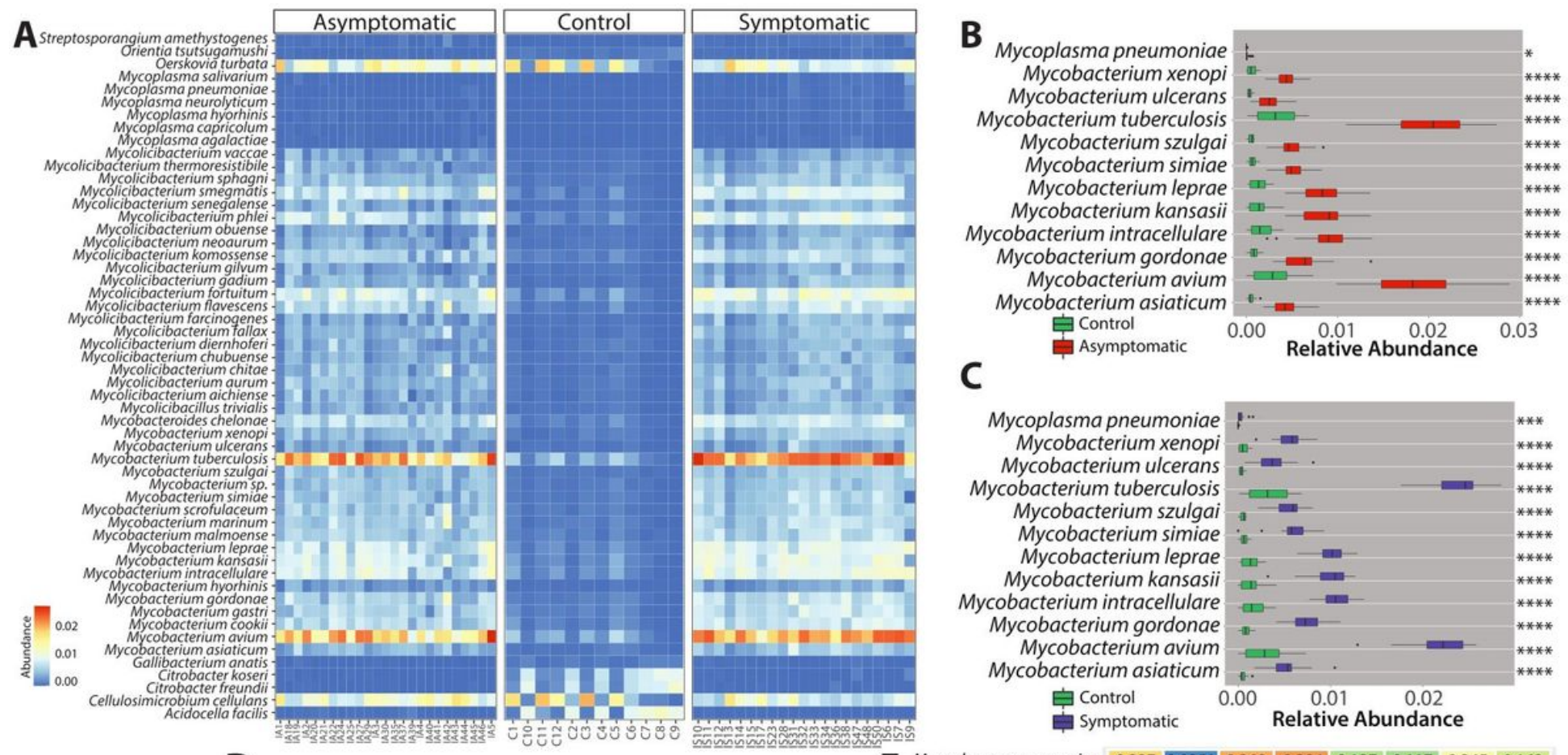

C

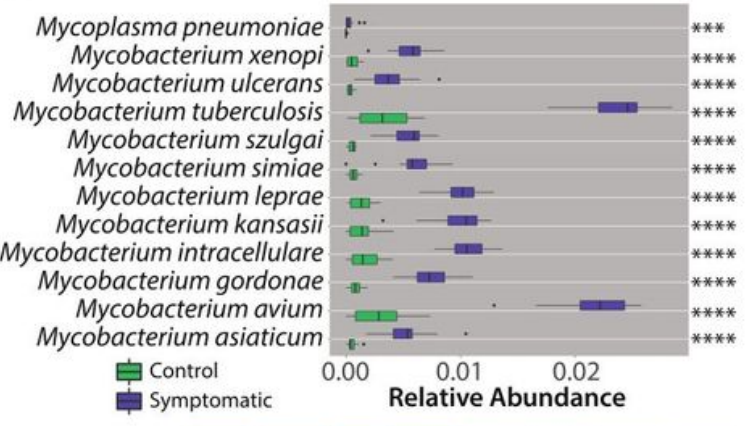

D

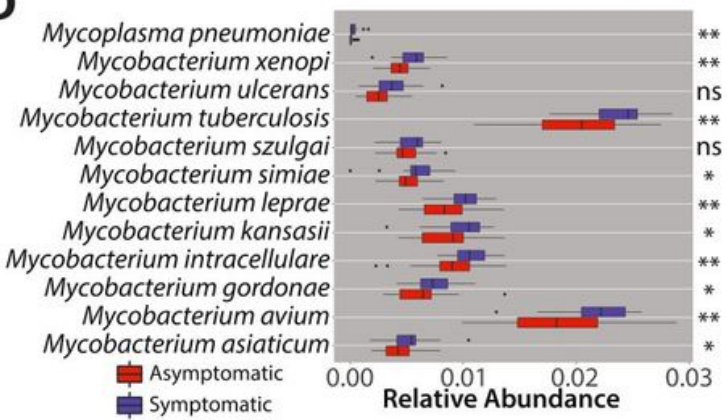

\begin{tabular}{|c|c|c|c|c|c|c|c|c|} 
E Mycoplasma pneumoniae & 0.337 & 0.0251 & 0.369 & 0.384 & 0.127 & 0.117 & 0.245 & 0.163
\end{tabular} \begin{tabular}{|l|l|l|l|l|l|l|l|l|}
\hline Mycobacterium xenopi & 0.3 & 0.235 & 0.258 & 0.388 & 0.123 & 0.203 & 0.29 & 0.382 \\
\hline
\end{tabular}

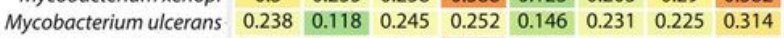
\begin{tabular}{|l|l|l|l|l|l|l|l|l|}
\hline Mycobacterium tuberculosis & 0.256 & 0.348 & 0.264 & 0.483 & 0.091 & 0.22 & 0.28 & 0.349
\end{tabular} \begin{tabular}{ll|l|l|l|l|l|l|l|l|} 
Mycobacterium szulgai & 0.248 & 0.164 & 0.217 & 0.266 & 0.178 & 0.265 & 0.24 & 0.332
\end{tabular} \begin{tabular}{|l|l|l|l|l|l|l|l|l|l|l|}
\hline Mycobacterium simiae & 0.193 & 0.238 & 0.264 & 0.406 & 0.21 & 0.265 & 0.223 & 0.34 \\
\hline
\end{tabular} \begin{tabular}{|l|l|l|l|l|l|l|l|l|l|} 
Mycobacterium leprae & 0.241 & 0.42 & 0.153 & 0.363 & 0.146 & 0.175 & 0.388 & 0.308 \\
\hline
\end{tabular} \begin{tabular}{ll|l|l|l|l|l|l|l|l|l} 
Mycobacterium kansasii & 0.182 & 0.4 & 0.179 & 0.424 & 0.0989 & 0.214 & 0.248 & 0.367
\end{tabular} \begin{tabular}{|l|l|l|l|l|l|l|l|l|l|}
\hline Mycobacterium gordonae & 0.238 & 0.303 & 0.34 & 0.39 & 0.178 & 0.243 & 0.211 & 0.326 \\
\hline
\end{tabular}

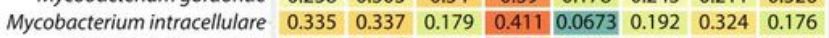
\begin{tabular}{|l|l|l|l|l|l|l|l|l|}
\hline Mycobacterium avium & 0.315 & 0.374 & 0.21 & 0.427 & 0.091 & 0.203 & 0.361 & 0.329
\end{tabular} \begin{tabular}{lll|l|l|l|l|l|l|l|l} 
Mycobacterium asiaticum & 0.312 & 0.189 & 0.299 & 0.393 & 0.154 & 0.214 & 0.201 & 0.275
\end{tabular}

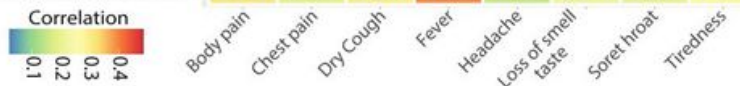

\section{Figure 6}

Relative abundance and species-specific OTUs reveal Mycobacterium spp. and Mycoplasma as key opportunistic pathogens. (A) Heatmap showing the taxonomic distribution of 54 species obtained from 12 genus based on LDA scores. (B-D) Boxplots showing relative abundance of opportunistic pathogens (Statistical parameters (Wilcoxon Rank test): ns: $p=>0.05,821 * p<=0.05, * \star p<=0.01, * \star * p<=0.001$, $\star \star \star \star ~ p<=0.0001$ ) in C vs. IA, C vs. IS, and IA vs. IS groups. (E) Spearman correlation showing relationship between opportunistic pathogenic bacteria and patient's clinical symptoms.

\section{Supplementary Files}

This is a list of supplementary files associated with this preprint. Click to download. 
- SupplementalTable1.xlsx

- SupplementalTable2.xlsx

- SupplementalTable3.xIsx 